\title{
Visualizing extremal positive maps in unital and trace preserving form
}

\author{
Leif Ove Hansen and Jan Myrheim \\ Department of Physics, Norwegian University of Science and Technology, \\ N-7491 Trondheim, Norway
}

August 8, 2018

\begin{abstract}
We define an entanglement witness in a composite quantum system as an observable having nonnegative expectation value in every separable state. Then a state is entangled if and only if it has a negative expectation value of some entanglement witness. Equivalent representations of entanglement witnesses are as nonnegative biquadratic forms or as positive linear maps of Hermitian matrices. As reported elsewhere, we have studied extremal entanglement witnesses in dimension $3 \times 3$ by constructing numerical examples of generic extremal nonnegative forms. These are so complicated that we do not know how to handle them other than by numerical methods. However, the corresponding extremal positive maps can be presented graphically, as we attempt to do in the present paper. We understand that a positive map is extremal when the image of $\mathcal{D}$, the set of density matrices, fills out $\mathcal{D}$ maximally, in a certain sense. For the graphical presentation of a map we transform it to a standard form where it is unital and trace preserving. We present an iterative algorithm for the transformation, which converges rapidly in all our numerical examples and presumably works for any positive map. This standard form of an entanglement witness is unique up to unitary product transformations.
\end{abstract}

Keywords: Entanglement witnesses, positive maps, convex sets.

\section{Introduction}

The phenomenon of quantum entanglement [1, 2] is central to the development and understanding of quantum information theory and computation [3]. For a general bipartite mixed quantum state it is not known how to determine in an efficient way whether the state is entangled or separable. An experimental setup usually involves mixed quantum states, and the need to control, manipulate and quantify entanglement in such states is therefore of fundamental importance. The characterization of entangled states of a quantum system composed of two subsystems thus remains one of the key unsolved problems in the theory of quantum information. We will consider here only the finite dimensional case.

Entanglement of a quantum state may be revealed experimentally through negative expectation values of certain observables, called entanglement witnesses, that have positive expectation values in all separable states [4, 5]. The Choi-Jamiołkowski isomorphism relates the entanglement witnesses to positive maps on matrix algebras [6, 7]. In this way the study of entanglement witnesses is closely related to the study of positive maps. This study was pioneered by Størmer [8], who has recently given an extensive review [9]. 
In order to investigate the separability problem using positive maps we need to understand the structure of the set of positive maps. Unfortunately, this is a problem which seems just as difficult as the original separability problem. Since the set of positive maps is a compact convex set, a classification of the extremal positive maps would entail a classification of all positive maps. This is our motivation for studying the extremal positive maps.

\section{Outline of this paper}

The contents of the paper are organized in the following manner.

In section 2 we review some basic theory regarding positive maps and entanglement witnesses. We define the link between entanglement witnesses and positive maps, known as the Choi-Jamiołkowski isomorphism. An important class of positive maps, the completely positive maps, is defined. These maps are quantum operations, since they map quantum states into quantum states. The important distinction between decomposable and nondecomposable maps is made, and the relation to the Peres separability criterion [10] is shown. Furthermore, the unital and trace preserving properties of positive maps are defined, and the relation between these two properties is established. These two concepts are further discussed in Section 3. Finally, the expectation values of entanglement witnesses in pure product states are introduced. These are positive semidefinite biquadratic forms, the zeros of which play an important role. The importance of the zeros for the understanding of the extremal entanglement witnesses and the corresponding extremal positive maps is outlined.

In section 3 we argue that any entanglement witness may be transformed into a unital and trace preserving form through a product transformation. We use the fact that a map is trace preserving if and only if the transposed map is unital to define conditions on this product transformation. We then suggest an iteration scheme to solve the resulting equations, and we define and investigate the conditions under which this iteration scheme will converge.

Finally, in Section 4 we use the unital and trace preserving form of a generic extremal entanglement witness in the $3 \times 3$ system that we have produced numerically [11], to create various plots showing how the corresponding positive map acts. We present similar plots illustrating the action of the Choi-Lam extremal positive map. We also discuss briefly an example of extremal witnesses in dimension $2 \times 4$.

\section{Preliminary theory}

In this section we introduce our notation and review some mathematical background.

We write $H_{k}$ for the set of Hermitian $k \times k$ matrices, which is a real Hilbert space with the natural scalar product

$$
\langle X, Y\rangle=\operatorname{Tr}(X Y) \text {. }
$$

The mathematical description of a finite dimensional composite quantum system involves the complex tensor product $\mathbb{C}^{N}=\mathbb{C}^{m} \otimes \mathbb{C}^{n}$ and the real tensor product $H_{N}=H_{m} \otimes H_{n}$ with $N=m n$. We write the components of $\psi \in \mathbb{C}^{N}$ as $\psi_{I}=\psi_{i j}$ where

$$
I=1,2, \ldots, N \leftrightarrow i j=11,12, \ldots, 1 n, 21, \ldots, m n .
$$

The matrix elements of an $N \times N$ matrix $A$ are $A_{I K}=A_{i j ; k l}$ with $I \leftrightarrow i j$ and $K \leftrightarrow k l$. A product vector $\psi=\phi \otimes \chi$ has components $\psi_{i j}=\phi_{i} \chi_{j}$, and a product matrix $A=B \otimes C$ 
has components $A_{i j ; k l}=B_{i k} C_{j l}$. We define the partial transpose $A^{P}$ as the transpose with respect to the second factor in the tensor product,

$$
\left(A^{P}\right)_{i j ; k l}=A_{i l ; k j} \text {. }
$$

We use density matrices, positive matrices of unit trace, to represent physical states of the most general kind, so called mixed states. The set of all $N \times N$ density matrices we call $\mathcal{D}_{N}$, or $\mathcal{D}$ if we need not specify the dimension. It is a compact convex set of dimension $N^{2}-1$, completely determined by its extremal points, which are the pure states $\rho=\psi \psi^{\dagger}$ with $\psi \in \mathbb{C}^{N}$.

It is a remarkable fact that the partial transpose $\rho^{P}$ of a density matrix $\rho$ need not be positive definite. A density matrix with a positive partial transpose is called a PPT state, thus we define the set of PPT states as $\mathcal{P}=\mathcal{D} \cap \mathcal{D}^{P}$.

\subsection{The geometry of density matrices}

A density matrix $\rho \in \mathcal{D}_{N}$ lies on the boundary $\partial \mathcal{D}_{N}$ if it has rank $k<N$. Then it lies in the interior of a face $\mathcal{D}_{k}(\mathcal{U})$, which is the set of density matrices on a $k$ dimensional subspace $\mathcal{U} \subset \mathbb{C}^{N}$. Every face of $\mathcal{D}_{N}$ is of the type $\mathcal{D}_{k}(\mathcal{U})$. Thus $\partial \mathcal{D}_{N}$ consists of faces arranged in the same hierarchical structure as the lattice of subspaces of $\mathbb{C}^{N}$.

A $2 \times 2$ density matrix has the form

$$
\rho=\frac{1}{2}\left(\begin{array}{cc}
1+z & x-\mathrm{i} y \\
x+\mathrm{i} y & 1-z
\end{array}\right)
$$

with $x, y, z$ real and $x^{2}+y^{2}+z^{2} \leq 1$. Thus $\mathcal{D}_{2}$ is a three dimensional sphere, the Bloch sphere. The boundary states, with $x^{2}+y^{2}+z^{2}=1$, are the pure states.

The set $\mathcal{D}_{3}$ of $3 \times 3$ density matrices has dimension eight. Its boundary $\partial \mathcal{D}_{3}$ consists of a seven dimensional set of rank two matrices, and a four dimensional set of rank one matrices, the pure states. Every rank two state in $\mathcal{D}_{3}$ lies in the interior of a Bloch sphere $\mathcal{D}_{2}(\mathcal{U})$.

\subsection{Entanglement and entanglement witnesses}

A density matrix $\rho \in \mathcal{D}_{N}$ is separable if it is of the form

$$
\rho=\sum_{a} p_{a} \sigma_{a} \otimes \tau_{a}
$$

where $\sigma_{a} \in \mathcal{D}_{m}, \tau_{a} \in \mathcal{D}_{n}$, and $p_{a}>0, \sum_{a} p_{a}=1$. Its partial transpose is then positive. This fact, that $\mathcal{S} \subset \mathcal{P}$ where $\mathcal{S}$ is the set of separable states, provides an easy test for separability, known as the Peres criterion [10]. A density matrix is entangled if it is not separable. The separability problem, how to decide whether a given density matrix is separable or entangled, is difficult because it is difficult to recognize the entangled PPT states.

We define the dual set

$$
\mathcal{S}^{\circ}=\left\{A \in H_{N} \mid \operatorname{Tr}(A \rho) \geq 0 \quad \forall \rho \in \mathcal{S}\right\} .
$$

The dual of $\mathcal{S}^{\circ}$ is $\mathcal{S}$, thus $\rho$ is separable if and only if $\operatorname{Tr}(A \rho) \geq 0$ for every $A \in \mathcal{S}^{\circ}$. For this reason we call a matrix $A \in \mathcal{S}^{\circ}$ an entanglement witness. Note that we do not impose here 
the usual condition on an entanglement witness $A$ that it should have at least one negative eigenvalue, so that $\operatorname{Tr}(A \rho)<0$ for some $\rho \in \mathcal{D}_{N}$.

The convex set $\mathcal{S}$ is completely determined by its extremal points, which are the pure product states $\rho=\psi \psi^{\dagger}=\left(\phi \phi^{\dagger}\right) \otimes\left(\chi \chi^{\dagger}\right)$ with $\psi=\phi \otimes \chi$ and $\phi^{\dagger} \phi=\chi^{\dagger} \chi=1$, for which

$$
\operatorname{Tr}(A \rho)=\psi^{\dagger} A \psi=(\phi \otimes \chi)^{\dagger} A(\phi \otimes \chi)=\sum_{i, j, k, l} \phi_{i}^{*} \chi_{j}^{*} A_{i j ; k l} \phi_{k} \chi_{l} .
$$

Thus $A \in \mathcal{S}^{\circ}$ if and only if the biquadratic form

$$
f_{A}(\phi, \chi)=(\phi \otimes \chi)^{\dagger} A(\phi \otimes \chi)
$$

is non-negative definite. Since

$$
(\phi \otimes \chi)^{\dagger} A^{P}(\phi \otimes \chi)=\left(\phi \otimes \chi^{*}\right)^{\dagger} A\left(\phi \otimes \chi^{*}\right)
$$

we see that $A^{P} \in \mathcal{S}^{\circ}$ if and only if $A \in \mathcal{S}^{\circ}$.

\subsection{Maps}

A map, in the terminology used here, is a linear transformation $\mathbf{M}: H_{m} \mapsto H_{n}$ such that $Y=\mathbf{M} X$ when

$$
Y_{j l}=\sum_{i, k} M_{j l ; i k} X_{i k}
$$

The complex matrix elements $M_{j l ; i k}$ defining the map satisfy the relations $M_{j l ; i k}=\left(M_{l j ; k i}\right)^{*}$, so that the map preserves Hermiticity. The relation

$$
A_{i j ; k l}=M_{j l ; k i}
$$

defines a one to one correspondence between a Hermitian matrix $A \in H_{N}$ and a map $\mathbf{M}=$ $\mathbf{M}_{A}$. As we define the correspondence here it differs slightly from the well known ChoiJamiołkowski isomorphism, which associates the matrix $A$ with the map $X \mapsto \mathbf{M}_{A}\left(X^{T}\right)$.

It is useful to introduce matrices $E_{a} \in H_{m}$ and $F_{b} \in H_{n}$ that are basis vectors in the two Hilbert spaces. Then the map $\mathbf{M}$ is given by real matrix elements $M_{b a}$ such that

$$
\mathbf{M} E_{a}=\sum_{b} M_{b a} F_{b}
$$

We will choose the basis vectors to be orthonormal, then the matrix elements are

$$
M_{b a}=\left\langle F_{b}, \mathbf{M} E_{a}\right\rangle
$$

Furthermore, we will choose the unit matrices as basis vectors, $E_{0}=I_{m} / \sqrt{m}, F_{0}=I_{n} / \sqrt{n}$. Then the matrices $E_{a}$ for $a=1,2, \ldots, m^{2}-1$ and $F_{b}$ for $b=1,2, \ldots, n^{2}-1$ are traceless.

The transposed map $\mathbf{N}=\mathbf{M}^{T}: H_{n} \mapsto H_{m}$ may be defined in a basis independent way by the condition that

$$
\left\langle\mathbf{M}^{T} Y, X\right\rangle=\langle Y, \mathbf{M} X\rangle \quad \text { for all } \quad X \in H_{m}, Y \in H_{n} .
$$

It has matrix elements $N_{a b}=M_{b a}$, or $N_{i k ; j l}=M_{l j ; k i}=\left(M_{j l ; i k}\right)^{*}$. Thus transposition of the map $\mathbf{M}$ corresponds to transposition of the real matrix $M_{a b}$ and Hermitian conjugation of the complex matrix $M_{j l ; i k}$. 
As defined here, the maps $\mathbf{M}_{A}$ and $\mathbf{M}_{A}^{T}$ act on $X \in H_{m}$ and $Y \in H_{n}$ as follows,

$$
\mathbf{M}_{A} X=\operatorname{Tr}_{1}\left(A\left(X \otimes I_{n}\right)\right), \quad \mathbf{M}_{A}^{T} Y=\operatorname{Tr}_{2}\left(A\left(I_{m} \otimes Y\right)\right),
$$

where $\operatorname{Tr}_{1}$ and $\operatorname{Tr}_{2}$ denote the partial traces. The matrix $A$ may be expanded in terms of the basis vectors $E_{a} \otimes F_{b} \in H_{N}$ as

$$
A=\sum_{a, b} M_{b a} E_{a} \otimes F_{b}
$$

\subsection{Entanglement witnesses and positive maps}

By definition, a positive map $\mathbf{M}: H_{m} \mapsto H_{n}$ transforms positive matrices into positive matrices, that is, if $\rho \geq 0$ then $\mathbf{M} \rho \geq 0$.

In terms of the map $\mathbf{M}_{A}$ the biquadratic form introduced in Equation (8) may be written as

$$
f_{A}(\phi, \chi)=\chi^{\dagger} \mathbf{M}_{A}\left(\phi \phi^{\dagger}\right) \chi=\phi^{\dagger} \mathbf{M}_{A}^{T}\left(\chi \chi^{\dagger}\right) \phi .
$$

The condition that $f_{A}(\phi, \chi) \geq 0$ for all $\phi \in \mathbb{C}^{m}, \chi \in \mathbb{C}^{n}$ means that $\mathbf{M}_{A}\left(\phi \phi^{\dagger}\right)$ is a positive matrix for every $\phi \in \mathbb{C}^{m}$. Since the pure states $\phi \phi^{\dagger}$ are all the extremal points of $\mathcal{D}_{m}$, this is another way of saying that $\mathbf{M}_{A}$ is a positive map.

We conclude that $A$ is an entanglement witness if and only if $\mathbf{M}_{A}$ is a positive map. An equivalent condition is that $\mathbf{M}_{A}^{T}$ is a positive map. This correspondence between entanglement witnesses and positive maps places the positive maps in a central position in the theory of quantum entanglement.

\subsection{Completely positive maps}

An obvious condition on a physical map $\mathbf{M}: H_{m} \mapsto H_{n}$, transforming physical states into physical states, is that it should be positive. A less obvious condition is that it should be completely positive, so that every map of the form $\mathbf{I} \otimes \mathbf{M}: H_{k} \otimes H_{m} \mapsto H_{k} \otimes H_{n}$ is positive, where $\mathbf{I}: H_{k} \mapsto H_{k}$ is the identity map.

The most general form of a completely positive map is

$$
\mathbf{M}_{A} X=\sum_{a} V_{a} X V_{a}^{\dagger}
$$

with $n \times m$ matrices $V_{a}$. This form gives the following matrix elements of $A$,

$$
A_{i j ; k l}=\left(A^{P}\right)_{i l ; k j}=\sum_{a}\left(V_{a}\right)_{j k}\left(V_{a}\right)_{l i}^{*} .
$$

We see that the map $\mathbf{M}_{A}$ is completely positive if and only if $A^{P}$ is positive. It is well known that $A^{P}$ may be positive without $A$ being positive, because the transposition map $\mathbf{T} X=X^{T}$ on $H_{n}$ is positive but not completely positive.

A separable state $\rho$, as defined in Equation (5), remains positive under the application of positive maps to its local parts, thus

$$
(\mathbf{M} \otimes \mathbf{N}) \rho=\sum_{a} p_{a}\left(\mathbf{M} \sigma_{a}\right) \otimes\left(\mathbf{N} \tau_{a}\right)
$$

is a positive matrix when $\mathbf{M}: H_{m} \mapsto H_{k}$ and $\mathbf{N}: H_{n} \mapsto H_{l}$ are positive maps. In this way positive maps give necessary conditions for separability. 
Positive maps can also give sufficient conditions for separability [4]. This follows from the correspondence described above between entanglement witnesses and positive maps. For $\rho \in H_{N}=H_{m n}$ let us define $\sigma \in H_{m^{2}}$ as

$$
\sigma=\left(\mathbf{T} \otimes \mathbf{M}_{A}^{T}\right) \rho,
$$

where $A$ is an entanglement witness and $\mathbf{M}_{A}$ the corresponding positive map. In index notation we have that

$$
\sigma_{k a ; i b}=\sum_{j, l} A_{a l ; b j} \rho_{i j ; k l} .
$$

Clearly $\sigma$ is positive if $\rho$ is separable. The other way around, if $\sigma$ is positive for every entanglement witness $A$ we have that

$$
\operatorname{Tr}(A \rho)=\sum_{i, j, k, l} A_{k l ; i j} \rho_{i j ; k l}=\sum_{k, a, i, b} \delta_{k a} \sigma_{k a ; i b} \delta_{i b} \geq 0
$$

for every $A$, implying that $\rho$ is separable.

Note that this proof of sufficiency does not use the full condition that $\sigma$ should be positive, it uses only the condition that $\sigma$ should have a non-negative expectation value in the maximally entangled pure state (Bell state) $\delta_{i k} \in \mathbb{C}^{m^{2}}$. If we use the full positivity condition, one single positive map can in principle reveal the entanglement of many different states for which we would need many different entanglement witnesses. The PPT condition associated with the transposition map is a striking example, it is equivalent to all the conditions provided by a much larger set of entanglement witnesses.

The basic reason for the efficiency of maps in revealing entanglement is nonlinearity: the separability condition that a product $\mathbf{M} \otimes \mathbf{N}$ of positive maps should map $\rho$ into a positive matrix is highly nonlinear in $\rho$. An entanglement witness $A$, on the other hand, gives an inequality $\operatorname{Tr}(A \rho) \geq 0$ linear in $\rho$.

\subsection{Decomposable maps}

A positive map $\mathbf{M}: H_{m} \mapsto H_{n}$ is said to be decomposable if it can be written as

$$
\mathbf{M}=\mathbf{M}_{1}+\mathbf{M}_{2} \mathbf{T}_{m}
$$

where $\mathbf{M}_{1}$ and $\mathbf{M}_{2}$ are completely positive, and $\mathbf{T}_{m}$ is transposition on $H_{m}, 8,13$.

Since $\mathbf{M}_{A} \mathbf{T}_{m}=\mathbf{M}_{A^{P}}$ it follows that $\mathbf{M}_{A}$ is decomposable if and only if $A=B+C$ where $B$ and $C^{P}$ are both positive matrices. This decomposition of $A$ implies for every PPT state $\rho$ that

$$
\operatorname{Tr}(A \rho)=\operatorname{Tr}(B \rho)+\operatorname{Tr}(C \rho)=\operatorname{Tr}(B \rho)+\operatorname{Tr}\left(C^{P} \rho^{P}\right) \geq 0 .
$$

In particular, $A$ is an entanglement witness, and we call it a decomposable witness. It is not very useful as a witness, however, since it can not reveal the entanglement of an entangled PPT state.

It was shown by Woronowicz that all positive maps $\mathbf{M}: H_{m} \mapsto H_{n}$ with $N=m n \leq 6$ are decomposable [14. In these dimensions entangled PPT states therefore do not exist, and the Peres criterion is both necessary and sufficient for separability. By virtue of the Peres criterion, the development of practically useful separability criteria in dimensions $N \geq 8$ is a problem closely related to the understanding of the nondecomposable positive maps. 


\subsection{Unital and trace preserving maps}

A map $\mathbf{M}: H_{m} \mapsto H_{n}$ is unital if it maps the identity $I_{m} \in H_{m}$ to the identity $I_{n} \in H_{n}$, that is, if

$$
\sum_{i, k} M_{j l ; i k} \delta_{i k}=\sum_{i} M_{j l ; i i}=\delta_{j l} .
$$

It is trace preserving if $\operatorname{Tr}(\mathbf{M} X)=\operatorname{Tr} X$ for all $X \in H_{m}$, that is, if

$$
\sum_{j, i, k} M_{j j ; i k} X_{i k}=\sum_{i} X_{i i}
$$

which means that

$$
\sum_{j} M_{j j ; i k}=\delta_{i k}
$$

Thus the map $\mathbf{M}$ is trace preserving if and only if $\mathbf{M}^{T}$ is unital. A physical map should conserve probability, which means that it should be trace preserving.

In terms of the corresponding matrix $A \in H_{N}$ the condition for $\mathbf{M}_{A}$ to be unital is that

$$
\mathbf{M}_{A} I_{m}=\operatorname{Tr}_{1} A=I_{n}
$$

and the condition for $\mathbf{M}_{A}$ to be trace preserving is that

$$
\mathbf{M}_{A}^{T} I_{n}=\operatorname{Tr}_{2} A=I_{m}
$$

Equation (29) implies that $\operatorname{Tr} A=\operatorname{Tr} I_{n}=n$, whereas Equation (30) implies that $\operatorname{Tr} A=$ $\operatorname{Tr} I_{m}=m$. Thus the map may be both unital and trace preserving only if $m=n$. If $m \neq n$ the proper condition is rather that

$$
\mathbf{M}_{A} E_{0}=F_{0}, \quad \mathbf{M}_{A}^{T} F_{0}=E_{0},
$$

where $E_{0}=I_{m} / \sqrt{m}$ and $F_{0}=I_{n} / \sqrt{n}$. Since we regard normalization constants as unimportant we will abuse the language and call the map unital and trace preserving if Equation (31) holds, even when $m \neq n$.

In terms of the matrix elements $M_{b a}=\left\langle F_{b}, \mathbf{M}_{A} E_{a}\right\rangle$ the condition for unitality is that $M_{b 0}=\delta_{b 0}$, and the condition for trace preservation is that $M_{0 a}=\delta_{0 a}$. This means that a unital and trace preserving map $\mathbf{M}_{A}$ is generated by an entanglement witness of the form

$$
A=\frac{I_{N}}{\sqrt{N}}+\sum_{a=1}^{m^{2}-1} \sum_{b=1}^{n^{2}-1} M_{b a} E_{a} \otimes F_{b} .
$$

\subsection{Zeros of entanglement witnesses}

Since the conditions defining $A \in H_{N}$ as an entanglement witness are the infinite set of inequalities

$$
f_{A}(\phi, \chi) \geq 0 \quad \text { for all } \quad \phi \in \mathbb{C}^{m}, \chi \in \mathbb{C}^{n},
$$

it is clear that $A$ is a boundary point of $\mathcal{S}^{\circ}$ if and only if at least one of these inequalities is an equality. We call the pure product state $\psi_{0} \psi_{0}^{\dagger}$ with $\psi_{0}=\phi_{0} \otimes \chi_{0}$ and $\phi_{0}^{\dagger} \phi_{0}=\chi_{0}^{\dagger} \chi_{0}=1 \mathrm{a}$ zero of $A$ if

$$
f_{A}\left(\phi_{0}, \chi_{0}\right)=0
$$


Since a zero is a minimum of the non-negative function $f_{A}$, it follows that the first derivative in every direction at the zero must also vanish. These conditions amount to a set of equalities

$$
\left(\phi_{0} \otimes \chi_{0}\right)^{\dagger} A\left(\phi \otimes \chi_{0}\right)=\left(\phi_{0} \otimes \chi_{0}\right)^{\dagger} A\left(\phi_{0} \otimes \chi\right)=0 \quad \text { for all } \quad \phi \in \mathbb{C}^{m}, \chi \in \mathbb{C}^{n} .
$$

These equations may be regarded as linear constraints on the matrix $A$. It should be remembered that each equation is complex and is equivalent to two real equations, except that Equation (34) is real. A careful counting shows that one zero implies $2(m+n)-3$ real valued constraints on $A$.

If the second derivative in every direction is strictly positive, then the zero $\left(\phi_{0}, \chi_{0}\right)$ is a quadratic minimum, and there are no more constraints on $A$ from this zero. If the second derivative in some direction vanishes, then the third derivative in this direction must also vanish, and the zero is a quartic minimum. This imposes further constraints on $A$, which we do not detail here.

The distinction between quadratic and quartic zeros is important. Obviously, the quadratic zeros are isolated points. A quartic zero may be an isolated point, but it may also belong to a continuous set of zeros.

An entanglement witness $A$ is extremal if and only if it has so many zeros that all the constraints from all the zeros together determine $A$ uniquely up to a proportionality constant.

\subsection{Extremal positive maps}

An extremal entanglement witness $A$ is understood in terms of its zeros. It corresponds to an extremal positive map $\mathbf{M}_{A}$, and we have to understand what the zeros of the witness imply for the corresponding map.

Let $\left(\phi_{0}, \chi_{0}\right)$ be an isolated zero of $A$, and define $Y=\mathbf{M}_{A}\left(\phi_{0} \phi_{0}^{\dagger}\right), X=\mathbf{M}_{A}^{T}\left(\chi_{0} \chi_{0}^{\dagger}\right)$. We use the identities

$$
\chi_{0}^{\dagger} Y \chi_{0}=\phi_{0}^{\dagger} X \phi_{0}=\left(\phi_{0} \otimes \chi_{0}\right)^{\dagger} A\left(\phi_{0} \otimes \chi_{0}\right)=0 .
$$

Since $Y$ and $X$ are both positive matrices, it follows that

$$
Y \chi_{0}=0, \quad X \phi_{0}=0
$$

We also know that $Y$ and $X$ have no other zero vectors, otherwise the zero vectors would span subspaces of dimension two or higher, and the zero $\left(\phi_{0}, \chi_{0}\right)$ would not be isolated. Hence $Y$ has rank $n-1$ and $X$ has rank $m-1$.

To summarize, when $A$ is an extremal entanglement witness the corresponding maps $\mathbf{M}_{A}$ and $\mathbf{M}_{A}^{T}$ are extremal positive maps. An isolated zero $\left(\phi_{0}, \chi_{0}\right)$ of $A$ defines a rank one state $\phi_{0} \phi_{0}^{\dagger} \in \mathcal{D}_{m}$ mapped by $\mathbf{M}_{A}$ to a rank $n-1$ state in $\mathcal{D}_{n}$, and a rank one state $\chi_{0} \chi_{0}^{\dagger} \in \mathcal{D}_{n}$ mapped by $\mathbf{M}_{A}^{T}$ to a rank $m-1$ state in $\mathcal{D}_{m}$.

Thus, the zero of $A$ defines a point on the boundary of $\mathcal{D}_{m}$ which is mapped to a point on the boundary of $\mathcal{D}_{n}$, and a point on the boundary of $\mathcal{D}_{n}$ which is mapped by the transposed map to a point on the boundary of $\mathcal{D}_{m}$. We understand that the map $\mathbf{M}_{A}$ is extremal precisely because the image $\mathbf{M}_{A} \mathcal{D}_{m}$ inside $\mathcal{D}_{n}$ touches the boundary of $\mathcal{D}_{n}$ in as many points as possible.

\subsection{Rank one preserving maps}

The identity map I : $X \mapsto X$ and the transposition map $\mathbf{T}: X \mapsto X^{T}=X^{*}$ are two examples of extremal positive maps. They have the special property that they are rank one 
preservers, mapping pure states to pure states. They are extremal precisely because they map the boundary of $\mathcal{D}$ onto itself. They both preserve volume. In fact, the eigenvalues of $\mathbf{T}$ are \pm 1 , because $\mathbf{T}$ is its own inverse, $\mathbf{T}^{2}=\mathbf{I}$.

More generally, let $U$ be a unitary matrix and define $\mathbf{U}: X \mapsto U X U^{\dagger}$. Then $\mathbf{U}$ and $\mathbf{U T}$ are both rank one preservers, and they are extremal because they map the boundary of $\mathcal{D}$ onto itself. They also preserve volumes.

These maps and the corresponding entanglement witnesses are fundamentally different from the generic extremal witnesses we find numerically, which have only quadratic zeros, so that the corresponding maps map only a finite number of points from the boundary of $\mathcal{D}$ to the boundary, and map all other boundary points to the interior. An extremal map of this generic kind is contractive, it reduces volumes, since it maps $\mathcal{D}$ to a subset of itself.

\section{Transforming positive maps to unital and trace preserving form}

We argue now that every positive map $\mathbf{M}=\mathbf{M}_{A}$ where $A$ is an entanglement witness may be transformed into a unital and trace preserving form through a product transformation of the form [9]

$$
A \mapsto \widetilde{A}=(U \otimes V) A(U \otimes V)^{\dagger}
$$

where $U \in \mathrm{GL}(m, \mathbb{C})$ and $V \in \mathrm{GL}(n, \mathbb{C})$. Furthermore, we present here an efficient iteration procedure for doing the transformation numerically.

A product transformation of this kind preserves all the essential characteristics of $A$. For instance, if $A$ is extremal in $\mathcal{S}^{\circ}$ and non-decomposable, then so is $\widetilde{A}$, and a zero $\phi \otimes \chi$ of $A$ corresponds to a zero $\widetilde{\phi} \otimes \widetilde{\chi}$ of $\widetilde{A}$, where

$$
\widetilde{\phi}=\left(U^{\dagger}\right)^{-1} \phi, \quad \tilde{\chi}=\left(V^{\dagger}\right)^{-1} \chi .
$$

As we saw in Section 2.7 the result to be proved is that every entanglement witness $A$ may be transformed to a form as given in Equation (32). In [15] it was proved that every strictly positive density matrix may be transformed to such a form. But the theorem is actually valid more generally than it is stated there, since the proof is valid for every entanglement witness having no zeros, in other words, every witness lying in the interior of $\mathcal{S}^{\circ}$. Here we want to apply this type of transformation to extremal witnesses, which lie on the boundary $\partial \mathcal{S}^{\circ}$ and have a maximal number of zeros. What could in principle go wrong in the limit when the boundary is approached from the inside of $\mathcal{S}^{\circ}$ is that the transformation could become singular, but we find in practice that no such problems arise.

In terms of the transformed map $\widetilde{\mathbf{M}}=\mathbf{M}_{\widetilde{A}}$ the conditions to be fulfilled are that $\widetilde{\mathbf{M}} I_{m}=I_{n}$ and $\widetilde{\mathbf{M}}^{T} I_{n}=I_{m}$. We will assume here that $m=n$, otherwise the proper conditions would be that $\widetilde{\mathbf{M}}\left(I_{m} / \sqrt{m}\right)=I_{n} / \sqrt{n}$ and $\widetilde{\mathbf{M}}^{T}\left(I_{n} / \sqrt{n}\right)=I_{m} / \sqrt{m}$.

In index notation Equation (38) reads as follows,

$$
\widetilde{A}_{i j ; k l}=\sum_{a, b, c, d} U_{i a} V_{j b} A_{a b ; c d} U_{k c}^{*} V_{l d}^{*} .
$$

According to Equation (15) the transformation $Y=\widetilde{\mathbf{M}} X$ then reads as follows,

$$
Y_{j l}=\sum_{i, k} \widetilde{A}_{i j ; k l} X_{k i}=\sum_{i, k, a, b, c, d} V_{j b}\left(A_{a b ; c d}\left(U_{k c}^{*} X_{k i} U_{i a}\right)\right) V_{l d}^{*}
$$


or in the indexfree notation,

$$
Y=\widetilde{\mathbf{M}} X=V\left(\mathbf{M}\left(U^{\dagger} X U\right)\right) V^{\dagger}
$$

Similarly, the transformation $X=\widetilde{\mathbf{M}}^{T} Y$ reads

$$
X_{i k}=\sum_{j, l} \widetilde{A}_{i j ; k l} Y_{l j}=\sum_{j, l, a, b, c, d} U_{i a}\left(A_{a b ; c d}\left(V_{l d}^{*} Y_{l j} V_{j b}\right)\right) U_{k c}^{*}
$$

or

$$
X=\widetilde{\mathbf{M}}^{T} Y=U\left(\mathbf{M}^{T}\left(V^{\dagger} Y V\right)\right) U^{\dagger} .
$$

The conditions for $\widetilde{\mathbf{M}}$ to be unital and trace preserving are that

$$
\begin{aligned}
\widetilde{\mathbf{M}} I_{m} & =V\left(\mathbf{M}\left(U^{\dagger} U\right)\right) V^{\dagger}=I_{n}, \\
\widetilde{\mathbf{M}}^{T} I_{n} & =U\left(\mathbf{M}^{T}\left(V^{\dagger} V\right)\right) U^{\dagger}=I_{m} .
\end{aligned}
$$

Thus the problem to be solved is to find $U$ and $V$ such that

$$
\mathbf{M}\left(U^{\dagger} U\right)=\left(V^{\dagger} V\right)^{-1}, \quad \mathbf{M}^{T}\left(V^{\dagger} V\right)=\left(U^{\dagger} U\right)^{-1} .
$$

\section{Solution by iteration}

This problem can be solved in two steps. First we find positive Hermitian matrices $X=U^{\dagger} U$ and $Y=V^{\dagger} V$ solving the equations

$$
\mathbf{M} X=Y^{-1}, \quad \mathbf{M}^{T} Y=X^{-1} .
$$

Then we solve the equations

$$
U^{\dagger} U=X, \quad V^{\dagger} V=Y
$$

for $U$ and $V$. The general solutions are

$$
U=U_{1} U_{2}, \quad V=V_{1} V_{2}
$$

where $U_{1}$ and $V_{1}$ are arbitrary unitary matrices, and $U_{2}=\sqrt{X}, V_{2}=\sqrt{Y}$ are the uniquely defined positive Hermitian square roots, which we compute for example by diagonalizing $X$ and $Y$ and taking the square roots of the eigenvalues.

Equation (47) makes sense because the matrices $X=U^{\dagger} U$ and $Y=V^{\dagger} V$ are strictly positive as long as $U$ and $V$ are nonsingular, and the maps $\mathbf{M}$ and $\mathbf{M}^{T}$, as well as the inversions $X \mapsto X^{-1}$ and $Y \mapsto Y^{-1}$, transform strictly positive matrices into strictly positive matrices.

The method suggesting itself for solving Equation (47) is simply to iterate the equations. Given an approximate solution $X_{k}$ for $X$ we try to compute a better approximation $X_{k+1}$ by a series of four transformations,

$$
X_{k} \mapsto S_{k}=\mathbf{M} X_{k} \mapsto Y_{k}=S_{k}^{-1} \mapsto T_{k}=\mathbf{M}^{T} Y_{k} \mapsto X_{k+1}=T_{k}^{-1}
$$

We start the iterations for example with $X_{0}=I$. 
A sufficient condition for the convergence of $X_{k}$ to a unique limit $X$ is that each transformation $X_{k} \mapsto X_{k+1}$ is contractive (except in the direction along $X$ ). A small perturbation $\Delta X_{k}$ of $X_{k}$ transforms linearly as

$$
\Delta X_{k+1}=\mathbf{D}\left(\Delta X_{k}\right),
$$

where the linear map $\mathbf{D}$ is the derivative of the nonlinear transformation $X_{k} \mapsto X_{k+1}$. The transformation is contractive if all eigenvalues of $\mathbf{D}$ (except the special eigenvalue which must be close to 1) are smaller than one in absolute value. Now $\mathbf{D}$ is a composition of four linear maps,

$$
\mathbf{D}=\mathbf{D}_{4} \mathbf{M}^{T} \mathbf{D}_{2} \mathbf{M}
$$

where $\mathbf{D}_{2}$ and $\mathbf{D}_{4}$ are linearizations of the matrix inversions,

$$
\begin{aligned}
& \mathbf{D}_{2}\left(\Delta S_{k}\right)=-S_{k}^{-1}\left(\Delta S_{k}\right) S_{k}^{-1}, \\
& \mathbf{D}_{4}\left(\Delta T_{k}\right)=-T_{k}^{-1}\left(\Delta T_{k}\right) T_{k}^{-1} .
\end{aligned}
$$

The examples of extremal positive maps $\mathbf{M}$ and $\mathbf{M}^{T}$ that we have studied numerically are strongly contractive, and we find in practice that this is enough to ensure that $\mathbf{D}$ is contractive, with eigenvalues typically no larger than about 0.5 , even though $\mathbf{D}_{2}$ and $\mathbf{D}_{4}$ are not contractive.

We have used this iteration scheme on a large number of numerically produced extremal entanglement witnesses [11], and also on many non-extremal witnesses constructed as convex combinations of the extremal ones. Numerically our attempts, which are in the thousands, always converge, and it also appears that for a given witness $A$ the solution $X$ is unique, independent of the initial guess $X_{0}$.

\section{Visualization of positive maps}

We now specialize to the case $m=n=3, N=m n=9$. The set $\mathcal{D}=\mathcal{D}_{3}$ of normalized density matrices has dimension $3^{2}-1=8$. When $m=n$ a linear positive map from $H_{m}$ to $H_{n}$ may be transformed to a form where it is unital and trace preserving.

Given a unital and trace preserving positive map $\mathbf{M}: H_{3} \mapsto H_{3}$. It maps $\mathcal{D}$ into $\mathcal{D}$, and the maximally mixed state $I / 3$ to itself. We plot two dimensional planar sections in order to illustrate how the image $\mathbf{M} \mathcal{D}$ lies inside $\mathcal{D}$.

We present such plots here for two different examples of extremal positive maps. The first example corresponds to a randomly chosen generic extremal witness found in a numerical search. Such witnesses have only quadratic zeros. The second example is the Choi-Lam map, which is qualitatively different since the corresponding witness has only quartic zeros.

\subsection{Two dimensional sections through the set of density matrices}

In most of our plots we use three density matrices $\rho_{0}, \rho_{1}, \rho_{2} \in \mathcal{D}$ to define a plane $\mathcal{Z} \subset H_{3}$. We use $\rho_{0}$ as origin in the plane, and define coordinate axes

$$
B=a\left(\rho_{1}-\rho_{0}\right), \quad C=b\left(\rho_{2}-\rho_{0}\right)+c\left(\rho_{1}-\rho_{0}\right),
$$

with real constants $a, b, c$ chosen in some way to be specified later on. We will always choose $a>0$ and $b>0$. A matrix $X \in \mathcal{Z}$ is then specified by a coordinate pair $(x, y)$ as

$$
X=\rho_{0}+x B+y C .
$$


Note that $\operatorname{Tr} B=\operatorname{Tr} C=0$ because $\operatorname{Tr} \rho_{i}=1$ for $i=0,1,2$, hence $\operatorname{Tr} X=\operatorname{Tr} \rho_{0}=1$.

\section{Six types of sections}

Figure 1 is an example of a section through the set of density matrices, where we have chosen the maximally mixed state as the origin, that is, $\rho_{0}=I / 3$. The density matrices $\rho_{1}$ and $\rho_{2}$ helping to define the section are here chosen at random. By Equation (54) the $x$ axis goes in the direction from $\rho_{0}$ to $\rho_{1}$. For this plot we have chosen the constants $a, b, c$ in Equation (54) such that $B$ and $C$ are orthogonal unit vectors in $H_{3}$, that is,

$$
\operatorname{Tr}\left(B^{2}\right)=\operatorname{Tr}\left(C^{2}\right)=1, \quad \operatorname{Tr}(B C)=0 .
$$

This means that distances in our plot represent faithfully distances in $H_{3}$ as defined by the Hilbert-Schmidt metric. To locate numerically the boundary $\partial \mathcal{D}$ we write $x=r \cos \theta$, $y=r \sin \theta$, and with $\theta$ fixed we determine the largest value of $r$ such that the matrix $X=$ $\rho_{0}+x B+y C$ has no negative eigenvalues. The boundary is here a smooth curve consisting entirely of rank two states.

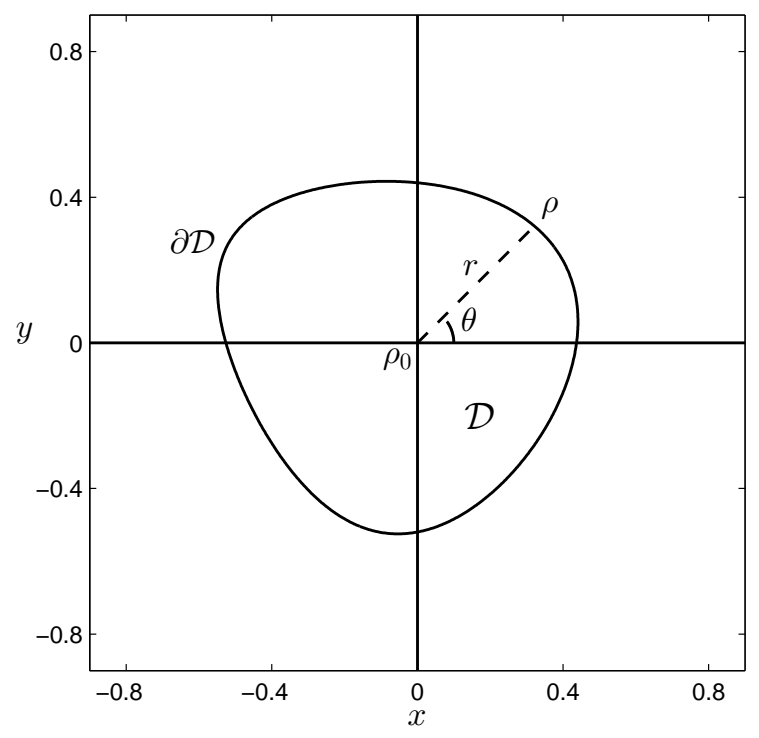

Figure 1: A two dimensional section through $\mathcal{D}$, the set of density matrices. The curve represents the boundary $\partial \mathcal{D}$ which consists of the states of less than full rank. For a given angle $\theta$ the distance $r$ is calculated numerically. The state $\rho_{0}=I / 3$ is the origin, and the state $\rho$ on the boundary has rank two. 

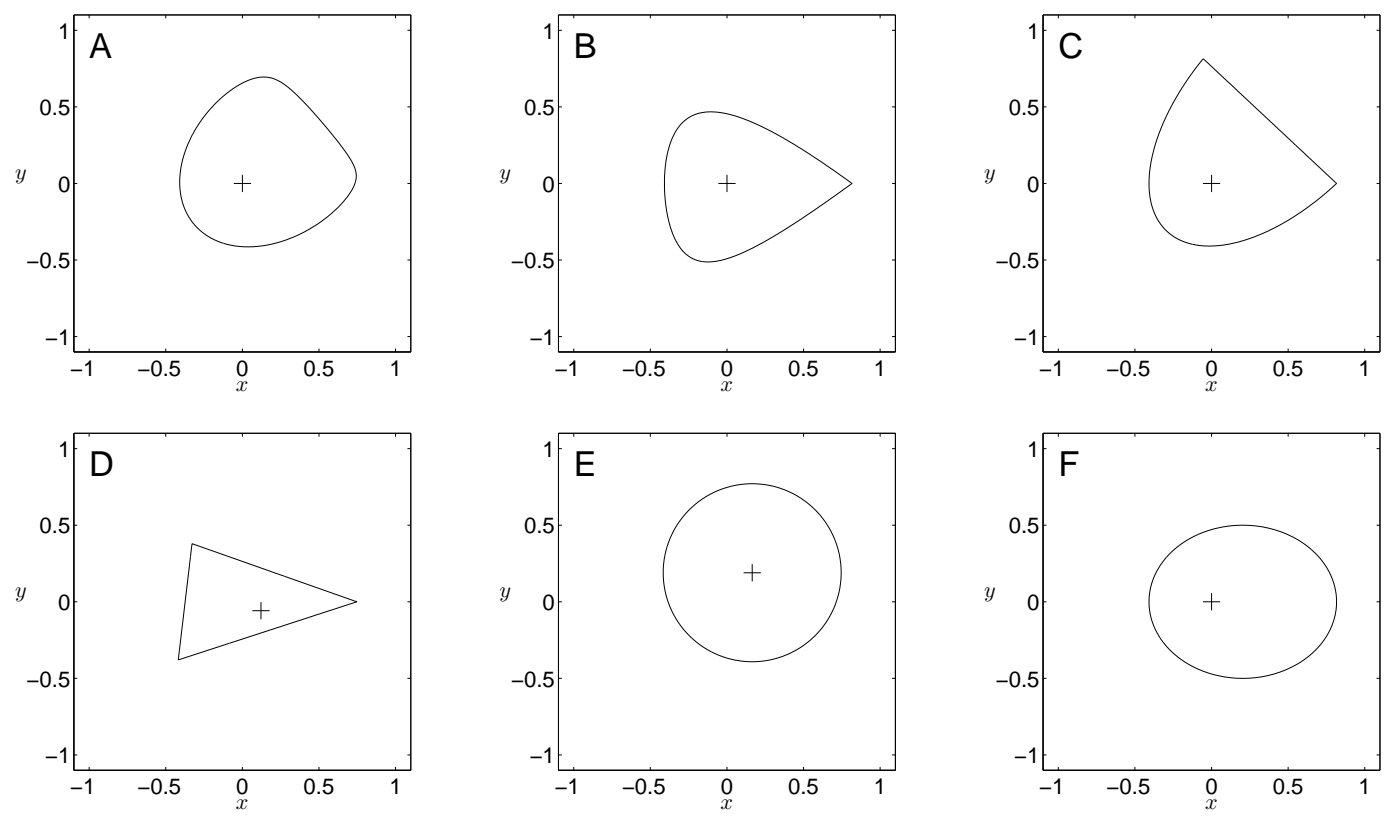

Figure 2: Six types of two dimensional sections through $\mathcal{D}$, as explained in the text.

Figure 2 shows six types of sections of $\mathcal{D}$. In the sections $\mathrm{A}, \mathrm{B}, \mathrm{C}$, and $\mathrm{F}$ the origin $\rho_{0}$ is the maximally mixed state $I / 3$, it is marked by a "+". In the sections $\mathrm{D}$ and $\mathrm{E}$ the "+" is the orthogonal projection of the maximally mixed state. All sections, except section E, cut through the interior of $\mathcal{D}$, so that the interior points in the sections are states of the full rank three.

Section A is of the same type as Figure 1, here $\rho_{1}$ and $\rho_{2}$ are chosen as random states of rank two, and the boundary is a smooth curve of rank two states.

In section $\mathrm{B}$ we choose $\rho_{1}$ to be a pure state, while $\rho_{2}$ is a random state of full rank. The pure state is seen in the plot as the single point on the boundary curve where the tangent direction is discontinuous. All other boundary points are states of rank two.

In section $\mathrm{C}$ both $\rho_{1}$ and $\rho_{2}$ are pure states, they are joined in the plot by a straight line of rank two states. The curved part of the boundary also consists of rank two states.

Section D is a simplex where the three corners are pure states $\rho_{k}=\phi_{k} \phi_{k}^{\dagger}$ for $k=1,2,3$ defined by linearly independent vectors $\phi_{k} \in \mathbb{C}^{3}$. The straight lines joining the pure states consist of rank two states. The origin is chosen as an even mix of the three pure states.

Section $\mathrm{E}$ is a two dimensional section through a three dimensional Bloch sphere contained in the boundary $\partial \mathcal{D}$. Like section $\mathrm{D}$ it is defined by three pure states, but in this case the three vectors in $\mathbb{C}^{3}$ are linearly dependent. In this section the interior points have rank two, and the boundary curve is a circle of pure states.

In section $\mathrm{F}$ we choose again a pure state $\rho_{1}=\phi_{1} \phi_{1}^{\dagger}$, but a section in which the boundary curve is smooth at this point. The three matrices defining the section are $\rho_{0}=I / 3, \rho_{1}$, and $\rho_{2}=\rho_{1}+D$, where the matrix $D$ is chosen in such a way that $\rho_{1}+\epsilon D$ for $\epsilon$ real is a pure state to first order in $\epsilon$, that is,

$$
\left(\phi_{1}+\epsilon \xi\right)\left(\phi_{1}+\epsilon \xi\right)^{\dagger}=\rho_{1}+\epsilon D+\mathcal{O}\left(\epsilon^{2}\right)
$$


for some $\xi \in \mathbb{C}^{3}$. We see that the proper choice is

$$
D=\phi_{1} \xi^{\dagger}+\xi \phi_{1}^{\dagger} .
$$

It is of no importance that $\rho_{2} \notin \mathcal{D}$. We choose $\xi$ orthogonal to $\phi_{1}$ in order to have $\operatorname{Tr} D=0$. The boundary points in this section, apart from $\rho_{1}$, are rank two states.

\section{Visualizing maps}

The positive map $\mathbf{M}$ maps the plane $\mathcal{Z}$ defined by the states $\rho_{i} \in \mathcal{D}$ with $i=0,1,2$ into the plane $\widetilde{\mathcal{Z}}=\mathbf{M} \mathcal{Z}$ defined by the states $\widetilde{\rho}_{i}=\mathbf{M} \rho_{i} \in \mathcal{D}$, in such a way that $X$ as given in Equation (55) is mapped into

$$
\widetilde{X}=\mathbf{M} X=\widetilde{\rho}_{0}+x \widetilde{B}+y \widetilde{C},
$$

with

$$
\widetilde{B}=a\left(\widetilde{\rho}_{1}-\widetilde{\rho}_{0}\right), \quad \widetilde{C}=b\left(\widetilde{\rho}_{2}-\widetilde{\rho}_{0}\right)+c\left(\widetilde{\rho}_{1}-\widetilde{\rho}_{0}\right) .
$$

It is an important point that $\operatorname{Tr}(\widetilde{B})=\operatorname{Tr}(\widetilde{C})=0$. This follows because we require the map $\mathbf{M}$ to be trace preserving.

The constants $a, b, c$ are the same here as in Equation (54). We choose them now to have such values that $\widetilde{B}$ and $\widetilde{C}$ are orthogonal unit vectors, that is,

$$
\operatorname{Tr}\left(\widetilde{B}^{2}\right)=\operatorname{Tr}\left(\widetilde{C}^{2}\right)=1, \quad \operatorname{Tr}(\widetilde{B} \widetilde{C})=0 .
$$

The motivation for this choice is that we want our plots of the coordinates $(x, y)$ to represent faithfully the distances in the image plane $\widetilde{\mathcal{Z}}$ of the map $\mathbf{M}$. These values for $a, b, c$ will obviously not, as a rule, be the same values that make $B$ and $C$ orthogonal unit vectors. Thus our $(x, y)$ plot will be a distorted representation of the plane $\mathcal{Z}$. The distortion is of course due to the map $\mathbf{M}$ between the two planes.

To summarize, in one and the same plot the coordinate pair $(x, y)$ represents both the matrix $X \in \mathcal{Z}$, given by Equation (55), and the matrix $\widetilde{X}=\mathbf{M} X \in \widetilde{\mathcal{Z}}$, given by Equation (59). Note that our definition of the axes $B, C$ and $\widetilde{B}, \widetilde{C}$ is such that $\widetilde{\rho}_{1}$ always has coordinates $y=0$ and $x>0$ in the plots, whereas $\widetilde{\rho}_{2}$ has $y>0$ but in general $x \neq 0$.

We plot the boundary of $\mathcal{D}$ in $\widetilde{\mathcal{Z}}$ by a full drawn curve, and the boundary of $\mathcal{D}$ in $\mathcal{Z}$ by a dashed curve. We calculate these boundaries by the method already described. That is, we write $x=r \cos \theta, y=r \sin \theta$. For a fixed value of $\theta$ we determine $r_{1}$ as the largest value of $r$ such that $X$ has no negative eigenvalue, this defines a point $\left(x_{1}, y_{1}\right)$ on the boundary of $\mathcal{D}$ in $\mathcal{Z}$, or equivalently, on the boundary of $\mathbf{M} \mathcal{D}$ in $\widetilde{\mathcal{Z}}$. And we determine $r_{2}$ as the largest value of $r$ such that $\widetilde{X}$ has no negative eigenvalue, this defines a point $\left(x_{2}, y_{2}\right)$ on the boundary of $\mathcal{D}$ in $\widetilde{\mathcal{Z}}$.

\subsection{One example: A generic extremal non-decomposable positive map}

In dimensions $m=n=3, N=m n=9$, we have constructed numerically extremal entanglement witnesses by random searches [11]. By definition, extremal witnesses found in random searches are generic.

The generic extremal witnesses we find have only quadratic zeros. Recall that an extremal witness is uniquely determined by its zeros. Since the number of constraints on the witness 
from one quadratic zero is $2(m+n)-3=9$, and the number of independent constraints must be $N^{2}-1=80$, at least nine zeros are necessary to determine the witness uniquely. Most of our generic extremal witnesses have exactly nine quadratic zeros, giving a total of 81 constraints, of which 80 are then independent.

The plots presented here show the action of one unital and trace preserving map $\mathbf{M}=$ $\mathbf{M}_{A}$, where $A$ is the transformed version, as described in Section 3, of a randomly selected generic extremal entanglement witness from the sample described in [11. The zeros of the transformed witness $A$ are $\psi_{i}=\phi_{i} \otimes \chi_{i}$ with $i=1,2, \ldots, 9$.

The "+" in each plot represents the orthogonal projection of the maximally mixed state $I / 3$ on the image plane $\widetilde{\mathcal{Z}}=\mathbf{M} \mathcal{Z}$. Since we use unital maps, if we choose $\rho_{0}=I / 3$ then $\widetilde{\rho}_{0}=\mathbf{M} \rho_{0}=\rho_{0}$ and the "+" is at the origin of the plot. The solid curve in each plot represents the boundary $\partial \mathcal{D}$ in the image plane $\widetilde{\mathcal{Z}}$. The dashed curve represents the image under $\mathbf{M}$ of $\partial \mathcal{D}$ in the plane $\mathcal{Z}$.

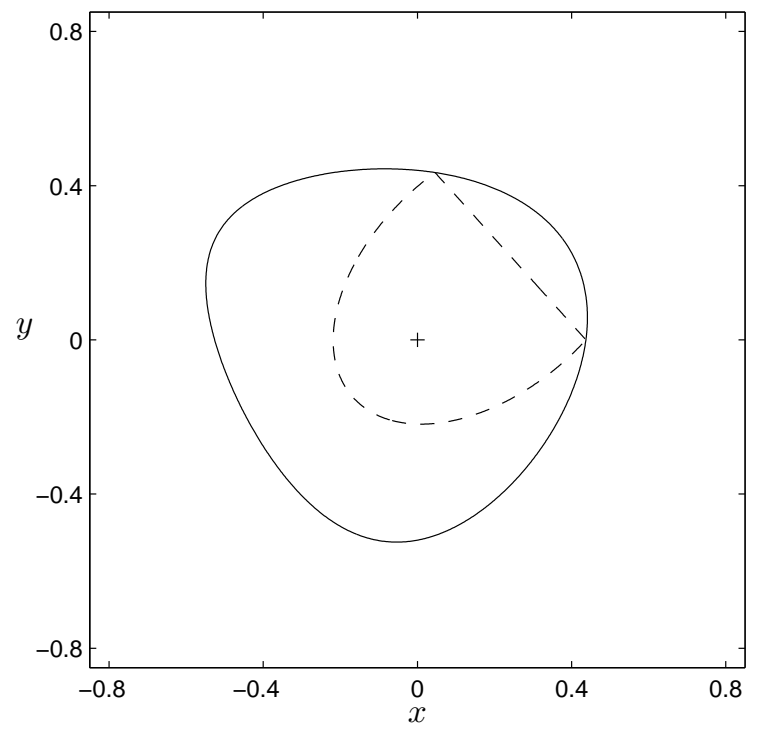

Figure 3: $\rho_{1}=\phi_{5} \phi_{5}^{\dagger}$ and $\rho_{2}=\phi_{6} \phi_{6}^{\dagger}$. The origin $\rho_{0}$ is the maximally mixed state $I / 3$.

Figure 3 shows a section with the maximally mixed state at the origin, that is, $\rho_{0}=\widetilde{\rho}_{0}=$ $I / 3$. We have chosen the plane $\mathcal{Z}$ to go through the pure states $\rho_{1}=\phi_{5} \phi_{5}^{\dagger}$ and $\rho_{2}=\phi_{6} \phi_{6}^{\dagger}$ corresponding to the arbitrarily chosen zeros number 5 and 6 of the extremal witness $A$. The section $\mathcal{Z} \cap \mathcal{D}$, shown by the dashed curve, is of the type $\mathrm{C}$ shown in Figure 2. The section $\widetilde{\mathcal{Z}} \cap \mathcal{D}$, shown by the full drawn curve, is of type A. The pure states $\rho_{1}$ and $\rho_{2}$ are mapped to rank two states $\widetilde{\rho}_{1}$ and $\widetilde{\rho}_{2}$, on the boundary $\partial \mathcal{D}$.

In Figure 4 a different origin is used, we have chosen $\rho_{0}$ as an even mix of the three pure states $\rho_{1}=\phi_{5} \phi_{5}^{\dagger}, \rho_{2}=\phi_{6} \phi_{6}^{\dagger}$, and $\rho_{3}=\phi_{7} \phi_{7}^{\dagger}$, which correspond to zeros of $A$ and are mapped to rank two states $\widetilde{\rho}_{1}, \widetilde{\rho}_{2}$, and $\widetilde{\rho}_{3}$. We observe that the projection of the maximally mixed state $I / 3$ is off center. This section $\mathcal{Z} \cap \mathcal{D}$ is of the type $\mathrm{D}$ shown in Figure 2, whereas the section $\widetilde{\mathcal{Z}} \cap \mathcal{D}$ is still of type A. Figures 5-9 show various types of sections, as explained in the figure captions. 


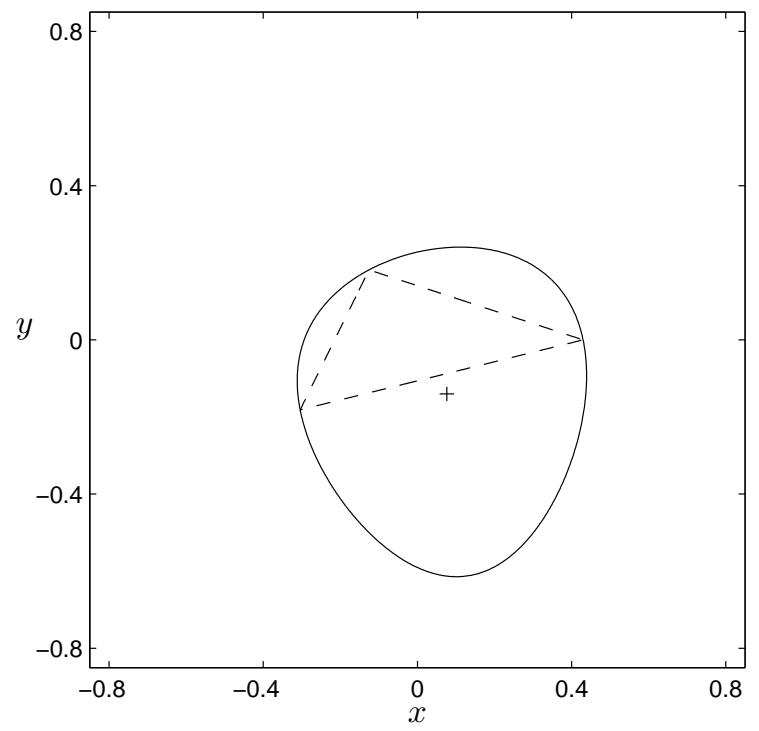

Figure 4: $\rho_{1}=\phi_{5} \phi_{5}^{\dagger}$ and $\rho_{2}=\phi_{6} \phi_{6}^{\dagger}$. The origin $\rho_{0}$ is an even mix of the pure states $\rho_{1}, \rho_{2}$, and $\rho_{3}=\phi_{7} \phi_{7}^{\dagger}$. The triangle is a face of an eight dimensional simplex defined by the nine zeros of the witness.

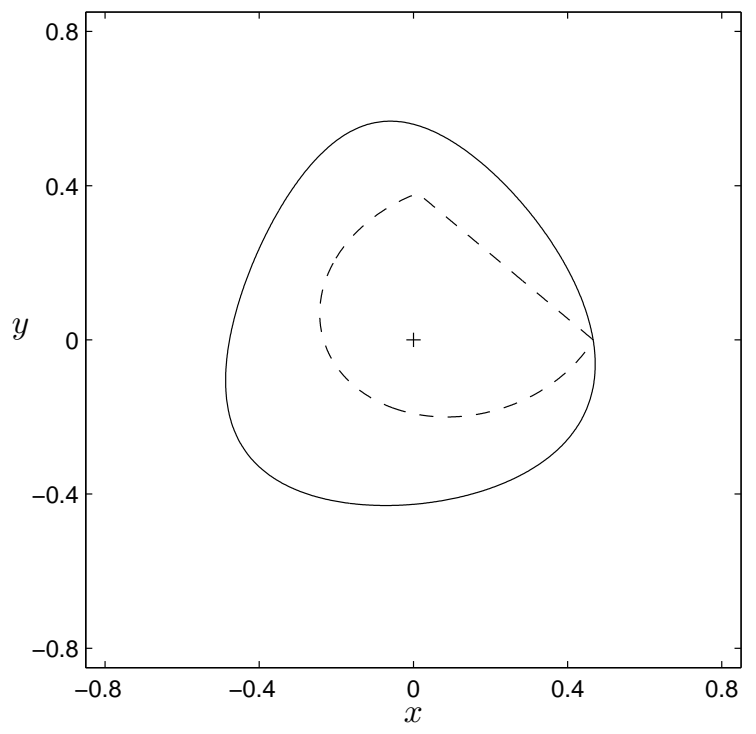

Figure 5: $\rho_{1}=\phi_{1} \phi_{1}^{\dagger}, \rho_{2}$ is a random pure state, and $\rho_{0}=I / 3$. Here $\widetilde{\rho}_{1}=\mathbf{M} \rho_{1}$ has rank two and lies on the boundary $\partial \mathcal{D}$, whereas $\widetilde{\rho}_{2}=\mathbf{M} \rho_{2}$ has full rank and lies in the interior of $\mathcal{D}$. The section $\mathcal{Z} \cap \mathcal{D}$ is of type $\mathrm{C}$. 


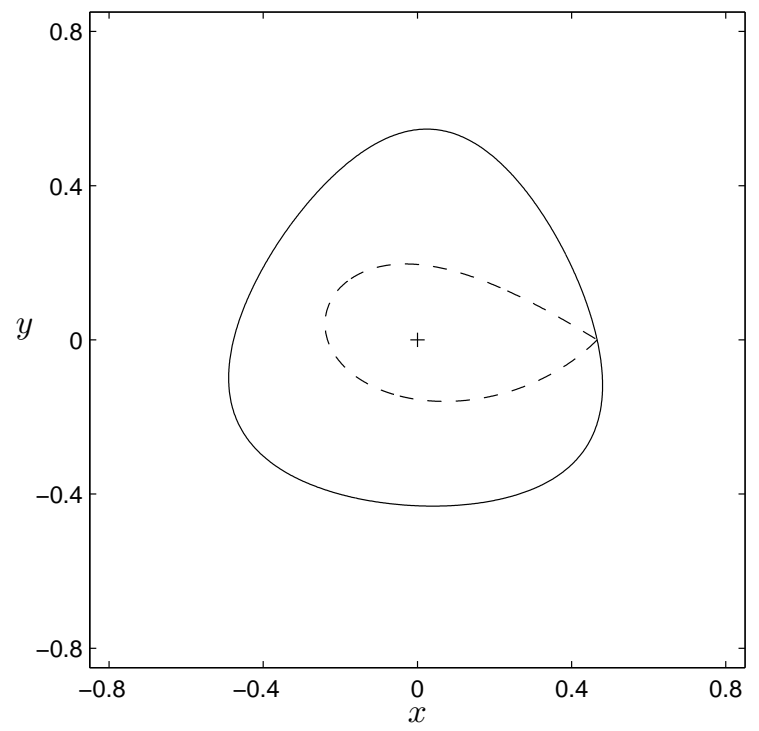

Figure 6: $\rho_{1}=\phi_{1} \phi_{1}^{\dagger}, \rho_{2}$ is a random state of rank three, and $\rho_{0}=I / 3$. Again $\rho_{1}$ is mapped to $\partial \mathcal{D}$ while $\rho_{2}$ is mapped to the interior of $\mathcal{D}$. The section $\mathcal{Z} \cap \mathcal{D}$ is of type $\mathrm{B}$.

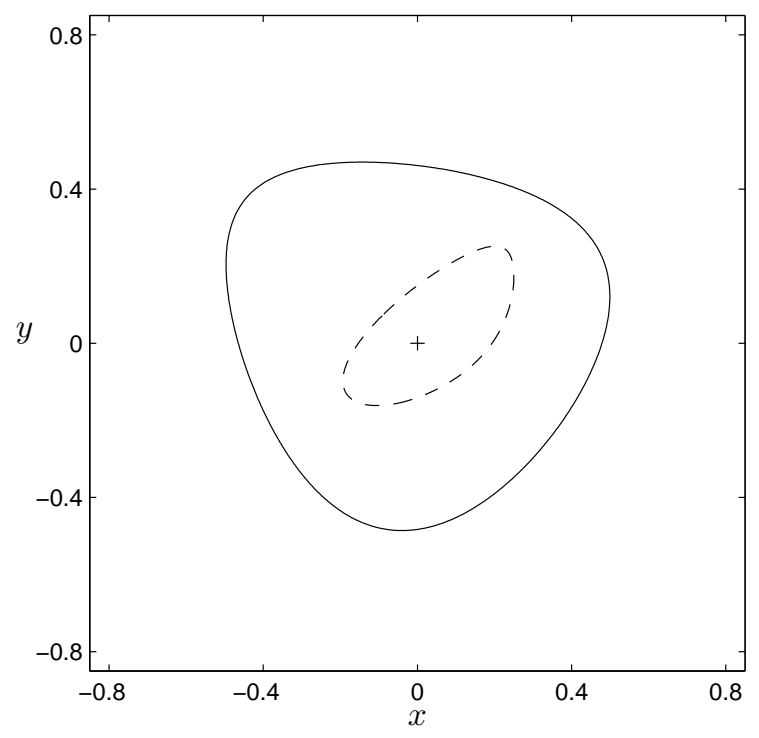

Figure 7: Both $\rho_{1}$ and $\rho_{2}$ are random states of rank three, and $\rho_{0}=I / 3$. Both sections in the plot are of type A. 


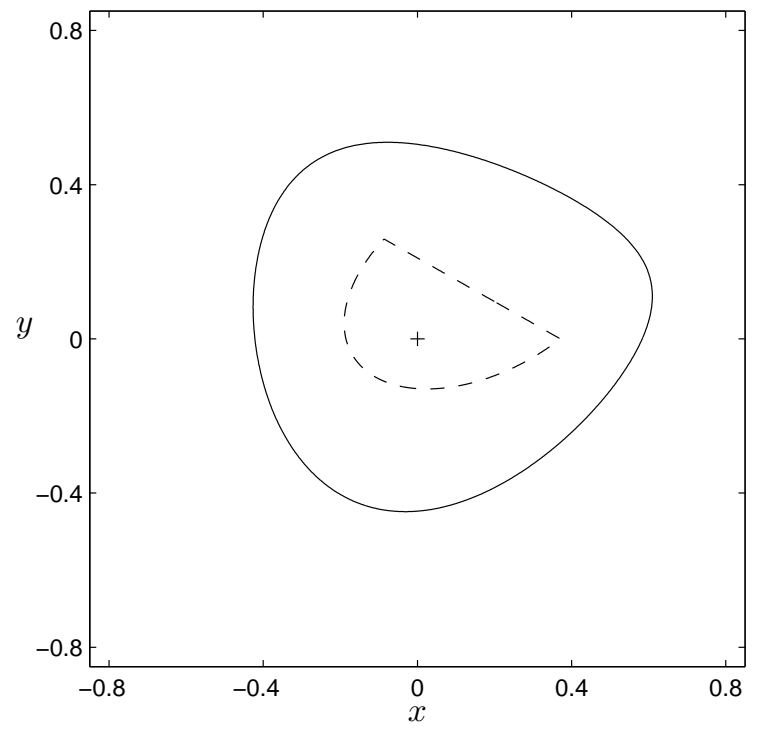

Figure 8: Here both $\rho_{1}$ and $\rho_{2}$ are random pure states, and $\rho_{0}=I / 3$. The section $\mathcal{Z} \cap \mathcal{D}$ is of type $\mathrm{C}$, it is mapped entirely to the interior of $\mathcal{D}$.

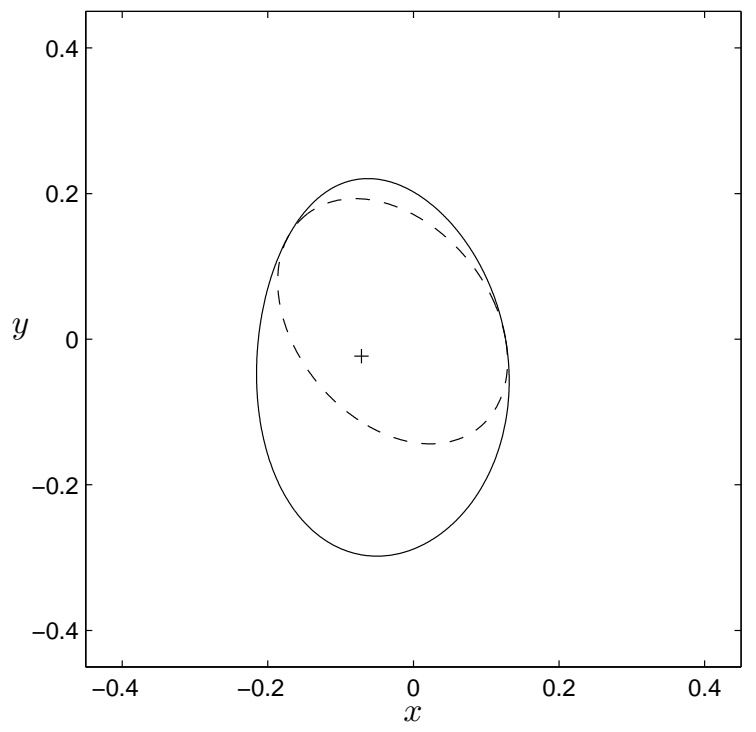

Figure 9: $\rho_{1}=\phi_{1} \phi_{1}^{\dagger}$ and $\rho_{2}=\phi_{2} \phi_{2}^{\dagger}$. The origin $\rho_{0}$ is an even mix of $\rho_{1}, \rho_{2}$, and $\rho_{3}=\xi \xi^{\dagger}$ where $\xi$ is a linear combination of $\phi_{1}$ and $\phi_{2}$. The section $\mathcal{Z} \cap \mathcal{D}$ is of type $\mathrm{E}$, it cuts through a Bloch sphere in $\partial \mathcal{D}$. Its circular shape is distorted by the map into an ellipse. The pure states $\rho_{1}$ and $\rho_{2}$ are mapped to rank two states, on $\partial \mathcal{D}$, whereas all the other pure states on the surface of the Bloch sphere are mapped to rank three states. The section $\widetilde{\mathcal{Z}} \cap \mathcal{D}$ is of type A, its shape is neither circular nor elliptical. 


\subsection{A second example: The Choi-Lam map}

The unital and trace preserving map

$$
\mathbf{M}: X \mapsto Y=\frac{1}{2}\left(\begin{array}{ccc}
X_{11}+X_{33} & -X_{12} & -X_{13} \\
-X_{21} & X_{11}+X_{22} & -X_{23} \\
-X_{31} & -X_{32} & X_{22}+X_{33}
\end{array}\right)
$$

was introduced by Choi and Lam in 1977 as the first example of an extremal non-decomposable positive map [7, 16]. It has been generalized to a one parameter family of extremal positive maps, but it is still one of the very few known analytical examples of such maps. It corresponds to the extremal entanglement witness $W$ with

$$
W^{P}=\left(\begin{array}{ccccccccc}
1 & \cdot & \cdot & \cdot & -1 & \cdot & \cdot & \cdot & -1 \\
\cdot & 1 & \cdot & \cdot & \cdot & \cdot & \cdot & \cdot & \cdot \\
\cdot & \cdot & \cdot & \cdot & \cdot & \cdot & \cdot & \cdot & \cdot \\
\cdot & \cdot & \cdot & \cdot & \cdot & \cdot & \cdot & \cdot & \cdot \\
-1 & \cdot & \cdot & \cdot & \cdot & \cdot & \cdot & \cdot & \cdot \\
\cdot & \cdot & \cdot & \cdot & \cdot & \cdot & \cdot & \cdot & -1 \\
\cdot & \cdot & \cdot & \cdot & \cdot & 1 & \cdot & \cdot & \cdot \\
\cdot & \cdot & \cdot & \cdot & \cdot & \cdot & 1 & \cdot & \cdot \\
-1 & \cdot & \cdot & \cdot & - & \cdot & \cdot & \cdot & \cdot \\
-1 & \cdot & \cdot & \cdot & 1
\end{array}\right)
$$

where for clarity the many zero entries are represented as dots. The witness $W$ has three isolated quartic zeros,

$$
e_{13}=e_{1} \otimes e_{3}, \quad e_{21}=e_{2} \otimes e_{1}, \quad e_{32}=e_{3} \otimes e_{2},
$$

where $e_{1}, e_{2}, e_{3}$ are the natural basis vectors in $\mathbb{C}^{3}$, and a continuum of quartic zeros $\phi \otimes \phi$ where

$$
\phi=\phi(\alpha, \beta)=e_{1}+\mathrm{e}^{\mathrm{i} \alpha} e_{2}+\mathrm{e}^{\mathrm{i} \beta} e_{3},
$$

and $\alpha, \beta \in \mathbb{R}$.

Equation (62) has a very simple geometrical interpretation. In the subspace of diagonal matrices there is a rotation by $60^{\circ}$ about the maximally mixed state, and in the subspace of off-diagonal matrices there is an inversion. There is also an overall contraction by a factor of one half.

Figure 10 shows the special section through $\mathcal{D}$ containing the diagonal matrices, including the maximally mixed state $\rho_{0}=I / 3$ and the three pure states

$$
\rho_{1}=e_{1} e_{1}^{\dagger}=\left(\begin{array}{ccc}
1 & 0 & 0 \\
0 & 0 & 0 \\
0 & 0 & 0
\end{array}\right), \quad \rho_{2}=e_{2} e_{2}^{\dagger}=\left(\begin{array}{ccc}
0 & 0 & 0 \\
0 & 1 & 0 \\
0 & 0 & 0
\end{array}\right), \quad \rho_{3}=e_{3} e_{3}^{\dagger}=\left(\begin{array}{lll}
0 & 0 & 0 \\
0 & 0 & 0 \\
0 & 0 & 1
\end{array}\right)
$$

corresponding to the three isolated zeros of the witness $W$. This section is mapped into itself by the map $\mathbf{M}=\mathbf{M}_{W}$, since

$$
\widetilde{\rho}_{1}=\left(\rho_{1}+\rho_{2}\right) / 2, \quad \widetilde{\rho}_{2}=\left(\rho_{2}+\rho_{3}\right) / 2, \quad \widetilde{\rho}_{3}=\left(\rho_{3}+\rho_{1}\right) / 2 .
$$

Figure 11 shows a section defined by the same pure states $\rho_{1}$ and $\rho_{2}$ as in Fig. 10, together with a random state of rank three. This section is not mapped into itself. 


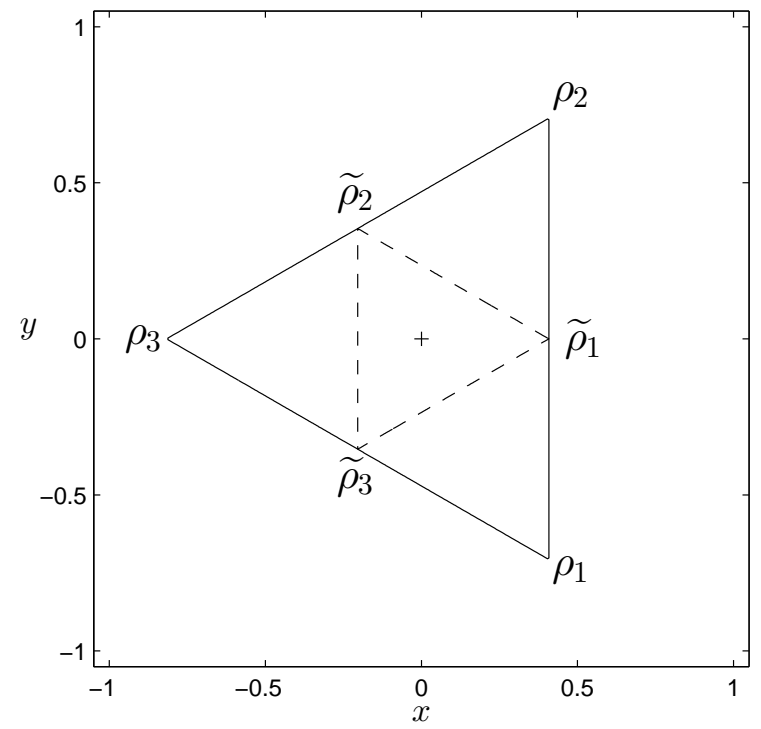

Figure 10: The Choi-Lam map in the plane of diagonal matrices. $\rho_{0}=I / 3, \rho_{1}=e_{1} e_{1}^{\dagger}$ and $\rho_{2}=e_{2} e_{2}^{\dagger}$.

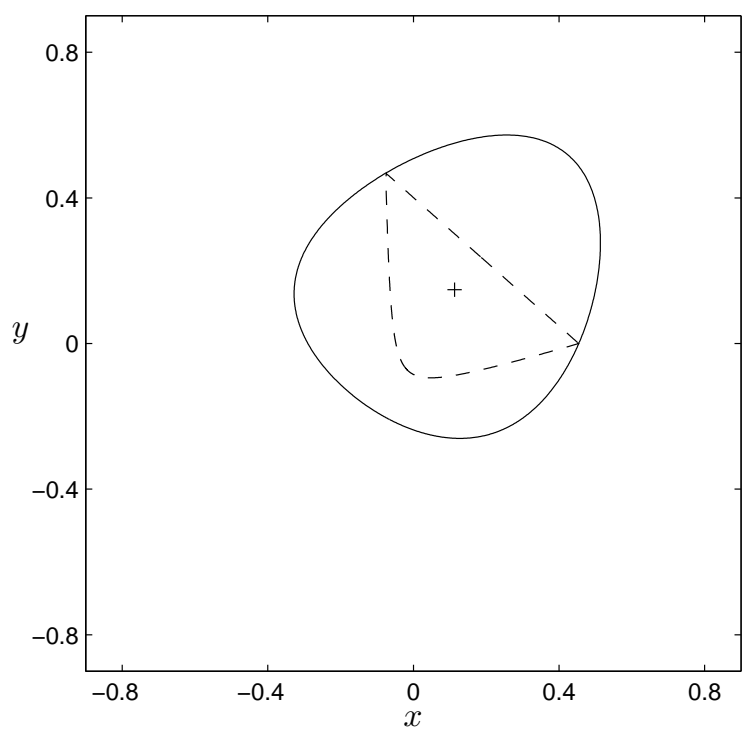

Figure 11: The Choi-Lam map. Another section with $\rho_{1}=e_{1} e_{1}^{\dagger}$ and $\rho_{2}=e_{2} e_{2}^{\dagger}$. The origin $\rho_{0}$ is chosen here as a random state of rank three, so the section $\mathcal{Z} \cap \mathcal{D}$ contains neither $e_{3} e_{3}^{\dagger}$ nor $I / 3$.

The continuous set of quartic zeros of the witness $W$, given in Equation (65), defines a 
two dimensional surface of pure states

$$
\rho(\alpha, \beta)=\frac{1}{3} \phi(\alpha, \beta)(\phi(\alpha, \beta))^{\dagger}=\rho_{0}+\sigma(\alpha, \beta)
$$

mapped by $\mathbf{M}$ to the boundary $\partial \mathcal{D}$. Here $\rho_{0}=I / 3$, and $\sigma(\alpha, \beta)$ is a completely off-diagonal matrix. We see directly from Equation (62) that

$$
\mathbf{M} \rho(\alpha, \beta)=\rho_{0}-\frac{1}{2} \sigma(\alpha, \beta)=\frac{1}{2}\left(3 \rho_{0}-\rho(\alpha, \beta)\right) .
$$

Thus the straight line through $\mathbf{M} \rho(\alpha, \beta)$ and $\rho_{0}$ contains the pure state $\rho(\alpha, \beta)$. This surface of pure states is curved, but we may choose our plane $\mathcal{Z}$ in such a way that it is tangent to the surface. Define for example

$$
\xi(\alpha)=\frac{\partial}{\partial \alpha} \phi(\alpha, \beta)=\mathrm{ie}^{\mathrm{i} \alpha} e_{2}
$$

Then the matrix

$$
D(\alpha, \beta)=\frac{\partial}{\partial \alpha} \rho(\alpha, \beta)=\frac{1}{3}\left(\xi(\alpha)(\phi(\alpha, \beta))^{\dagger}+\phi(\alpha, \beta)(\xi(\alpha))^{\dagger}\right)
$$

is a tangent to the surface such that

$$
\rho(\alpha, \beta)+\epsilon D(\alpha, \beta)=\rho(\alpha+\epsilon, \beta)+\mathcal{O}\left(\epsilon^{2}\right) .
$$

Figure 12 shows a section where we have chosen $\rho_{0}=I / 3$ and

$$
\begin{aligned}
& \rho_{1}=\rho(0,0)=\frac{1}{3}\left(e_{1}+e_{2}+e_{3}\right)\left(e_{1}+e_{2}+e_{3}\right)^{\dagger}, \\
& \rho_{2}=\rho_{1}+D(0,0)=\rho_{1}+\frac{\mathrm{i}}{3}\left(e_{2}\left(e_{1}+e_{3}\right)^{\dagger}-\left(e_{1}+e_{3}\right) e_{2}^{\dagger}\right) .
\end{aligned}
$$

It does not matter that $\rho_{2} \notin \mathcal{D}$. The coordinate axes as defined in Equation (54) are now

$$
B=a\left(\rho_{1}-\rho_{0}\right)=\frac{a}{3}\left(\begin{array}{lll}
0 & 1 & 1 \\
1 & 0 & 1 \\
1 & 1 & 0
\end{array}\right), \quad C=b\left(\rho_{2}-\rho_{1}\right)=b D(0,0)=\frac{b}{3}\left(\begin{array}{ccc}
0 & -\mathrm{i} & 0 \\
\mathrm{i} & 0 & \mathrm{i} \\
0 & -\mathrm{i} & 0
\end{array}\right) .
$$

According to Equation (62) this section is mapped into itself by a $180^{\circ}$ rotation and a scaling by $1 / 2$,

$$
B \mapsto \widetilde{B}=-\frac{1}{2} B, \quad C \mapsto \widetilde{C}=-\frac{1}{2} C .
$$

Both sections $\mathcal{Z} \cap \mathcal{D}$ and $\widetilde{\mathcal{Z}} \cap \mathcal{D}$ shown in Figure 12 are of type $\mathrm{F}$ in our classification. The numerical factors in Equation (75) making $\widetilde{B}$ and $\widetilde{C}$ orthonormal are $a=\sqrt{6}, b=3$. 


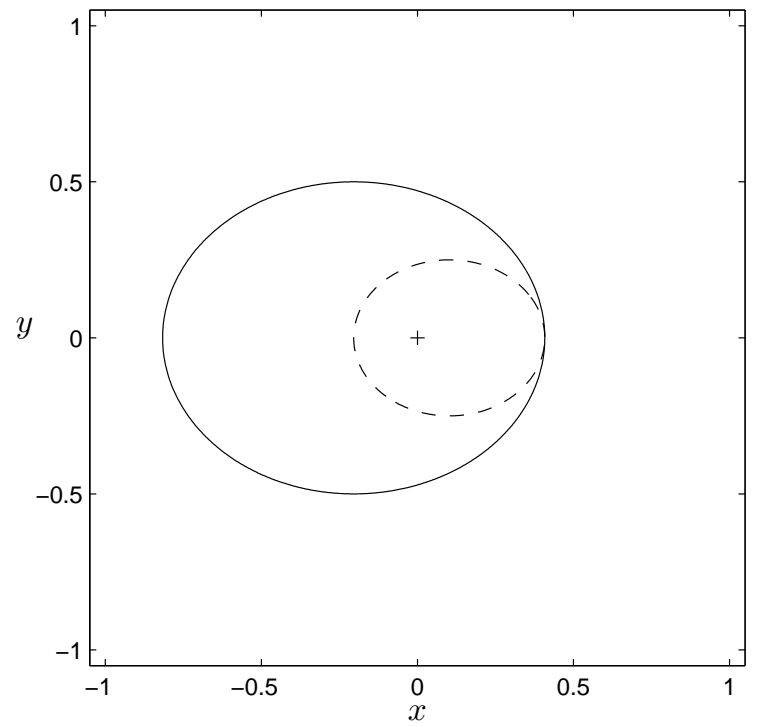

Figure 12: The Choi-Lam map. $\rho_{0}=I / 3, \rho_{1}=\rho(0,0)$, and $\rho_{2}=\rho_{1}+D(0,0)$, as defined in the text.

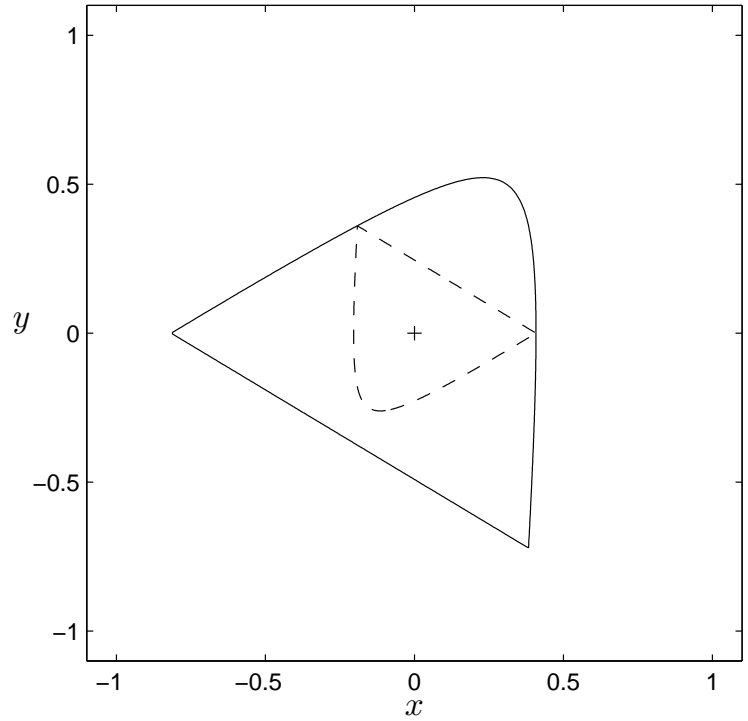

Figure 13: The Choi-Lam map. We use here $\rho_{0}=I / 3$ and two randomly chosen pure states $\rho_{1}=\phi_{1} \phi_{1}^{\dagger}, \rho_{2}=\phi_{2} \phi_{2}^{\dagger}$ corresponding to product vectors $\phi_{1} \otimes \phi_{1}^{*}, \phi_{2} \otimes \phi_{2}^{*}$ from the continuum of quartic zeros. 


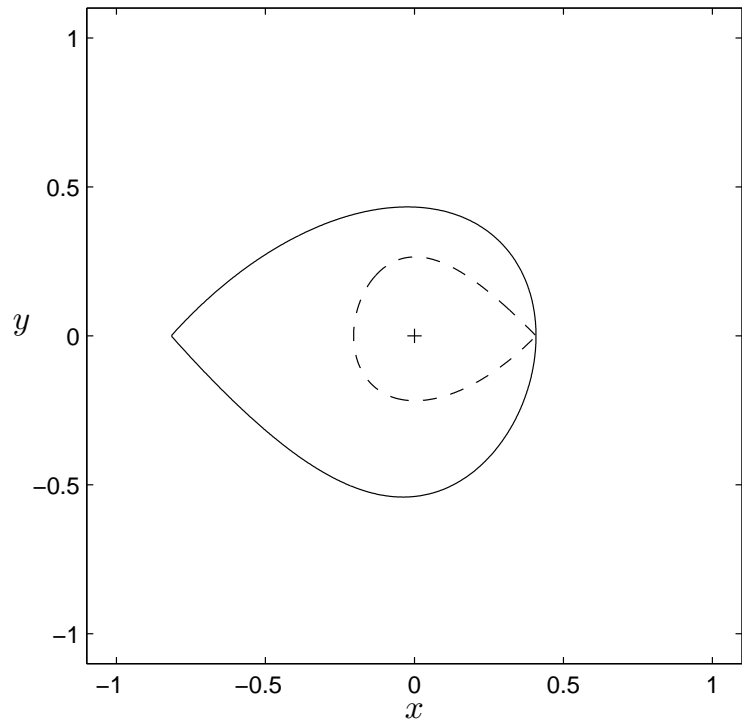

Figure 14: The Choi-Lam map. $\rho_{0}=I / 3, \rho_{1}=e_{1} e_{1}^{\dagger}$, and $\rho_{2}$ is a random state of rank three.

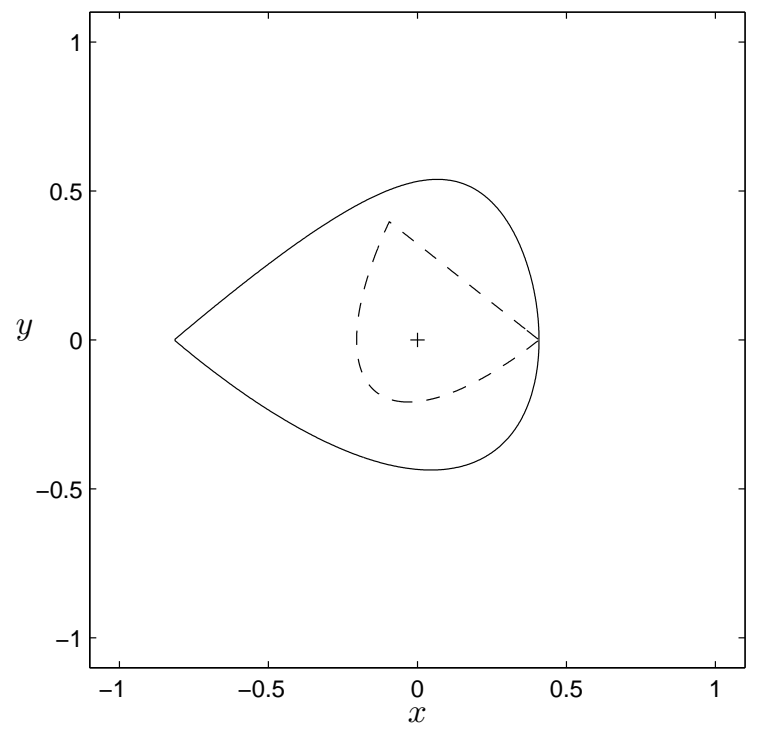

Figure 15: The Choi-Lam map. $\rho_{0}=I / 3, \rho_{1}=e_{1} e_{1}^{\dagger}$, and $\rho_{2}$ is a random pure state.

Figures 13-15 illustrate the Choi-Lam map by other sections, as explained in the figure captions. 


\subsection{A third example in $2 \times 4$ dimensions}

Our third example is based on the study of optimal witnesses by Lewenstein et al. [12. As an example they describe how to create optimal witnesses for proving the entanglement of the PPT states in $2 \times 4$ dimensions discovered by Paweł Horodecki [17]. We have chosen arbitrarily the parameter value $b=0.6$ and computed numerically the optimal witness as described in [12]. It has exactly eight zeros, all quadratic. It is not extremal, but is the centre of a two dimensional circular face of the set of normalized witnesses (normalized to have unit trace). The boundary of this face is a circle of quartic extremal witnesses, one of which defines the map described numerically in the Appendix. Note that this map is neither unital nor trace preserving.

This extremal witness has two separate continuous rings of zeros, which are all necessarily quartic, since they are not discrete. To a zero $\phi \otimes \chi$ with $\phi^{\dagger} \phi=\chi^{\dagger} \chi=1$ corresponds a density matrix

$$
\phi \phi^{\dagger}=\frac{1}{2}\left(\begin{array}{cc}
1+z & x-\mathrm{i} y \\
x+\mathrm{i} y & 1-z
\end{array}\right)
$$

with $x^{2}+y^{2}+z^{2}=1$. This rank one density matrix in $\mathcal{D}_{2}$ is mapped to a rank three density matrix in $\mathcal{D}_{4}$ with $\chi$ as an eigenvector of eigenvalue zero. Thus the image of the two rings on the boundary of $\mathcal{D}_{2}$ is where the image of $\mathcal{D}_{2}$ touches the inside of the boundary of $\mathcal{D}_{4}$.

The coordinates $x, y, z$ defining a zero are given by one parameter $\theta$ as follows. Let

$$
\begin{gathered}
a=0.1807362587783353, \quad b=0.047422228589395, \quad \theta_{0}=1.121090508802759, \\
s=\frac{a \cos \left(2 \theta+\theta_{0}\right)}{\cos \left(\theta-\theta_{0}\right)}, \quad t=\frac{-b s \pm \sqrt{1+s^{2}-b^{2}}}{1+s^{2}} \\
x=t \cos \theta, \quad y=t \sin \theta, \quad z=-b-t s .
\end{gathered}
$$

Note that $t \rightarrow 0$ and $t s \rightarrow-b \pm 1$ as $s \rightarrow \pm \infty$.

All the extremal quartic witnesses forming the circular boundary of the face have similar sets of zeros, it is only the parameter $\theta_{0}$ that varies as we go around the boundary. Any two of them have exactly eight zeros in common, corresponding to the special values $\theta=0$ and $\theta= \pm \pi / 3$, where $x, y, z$ are independent of $\theta_{0}$, and to $\theta=\theta_{0}+\pi / 2$, where $x=y=0, z= \pm 1$. Every witness in the interior of the face has exactly these eight zeros.

The Figures 16 to 18 show the zeros of the witness defining the map given in the Appendix. The Figures 19 to 21 show the same zeros, and in addition the zeros of two other extremal witnesses on the boundary of the same face. The zeros of different such extremal witnesses are found simply by changing the value of $\theta_{0}$. 


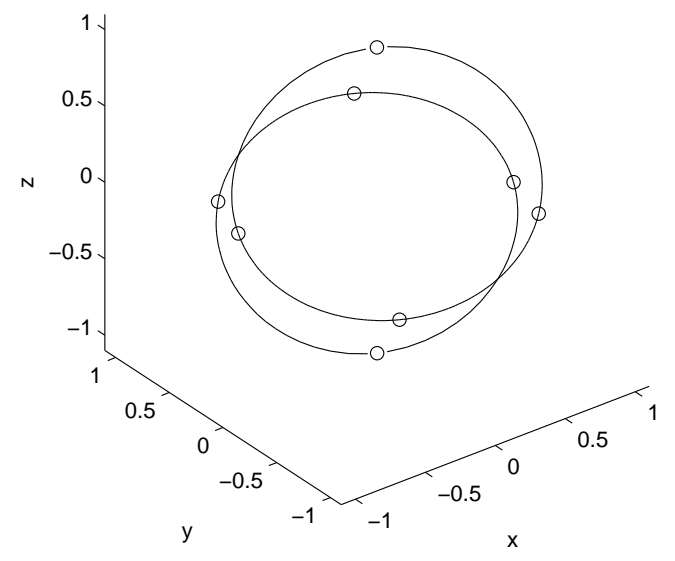

Figure 16: The two rings of zeros of the extremal witness in dimension $2 \times 4$, on the surface of the unit sphere. The eight zeros of the optimal witness are marked by small circles.

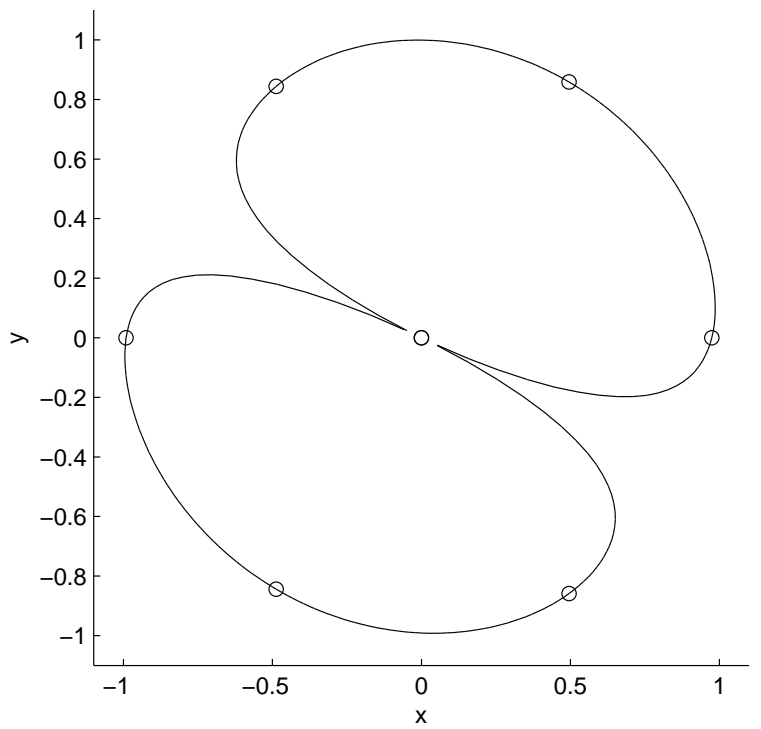

Figure 17: The $x y$ projection of Figure 16. Two of the eight special zeros are at the origin $x=y=0$, the six others are at angles $n \pi / 3$ from the $x$ axis with $n=0,1,2,3,4,5$. 


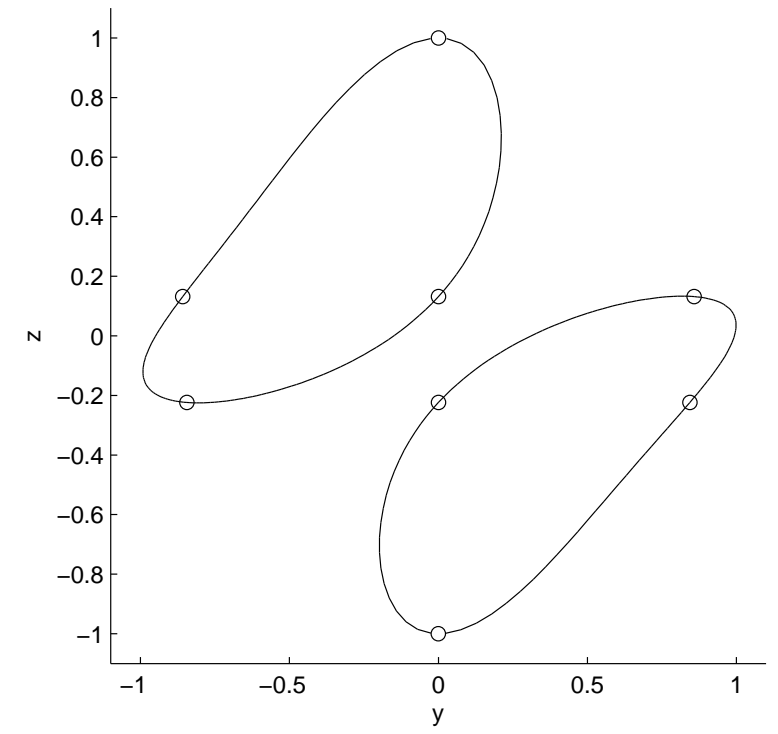

Figure 18: The $y z$ projection of Figure 16.

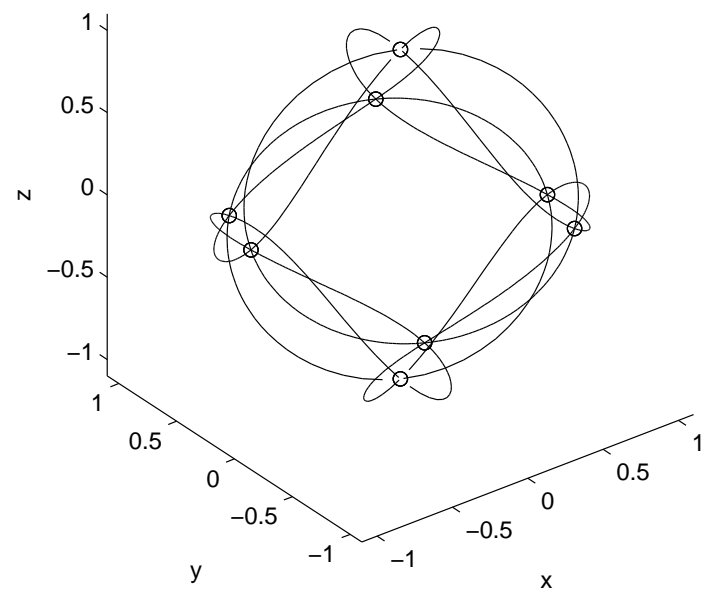

Figure 19: The same as Figure 16, but including the zeros of two other extremal witnesses with different values of $\theta_{0}$. 


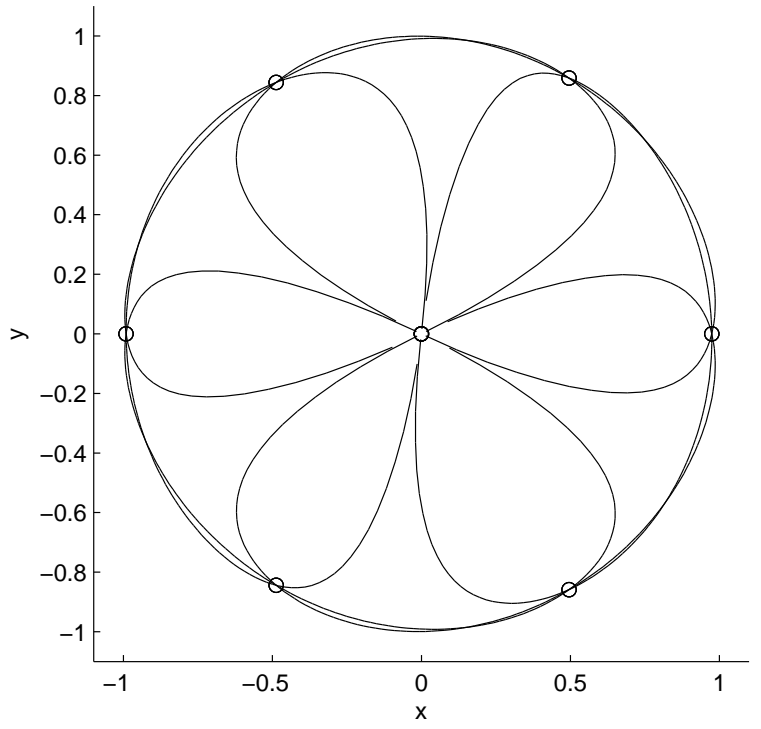

Figure 20: The $x y$ projection of Figure 19,

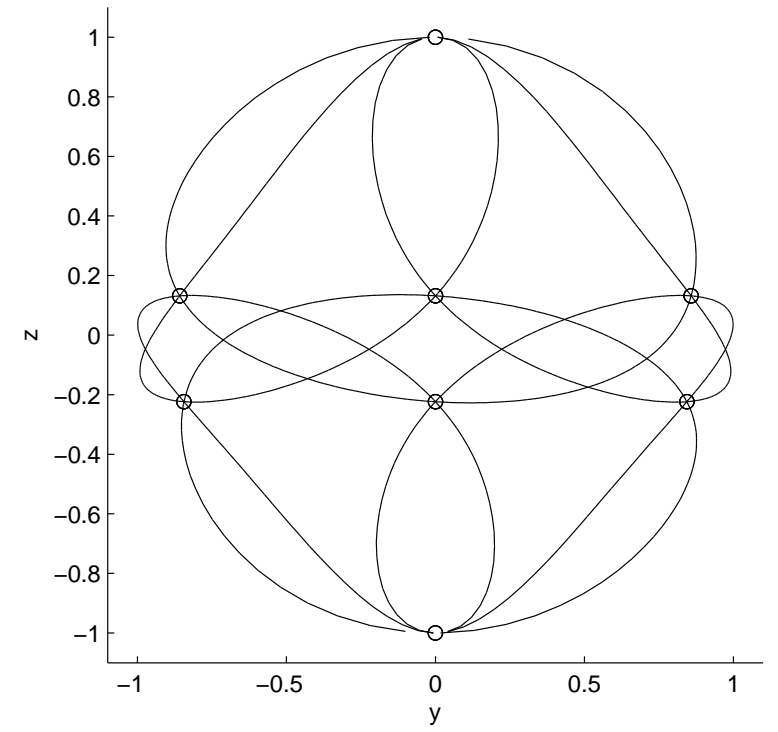

Figure 21: The $y z$ projection of Figure 19,

\section{$5 \quad$ Summary and conclusions}

The purpose of the work presented here has been to gain a more intuitive understanding of the geometry of positive maps, which are related to entanglement witnesses in bipartite 
quantum systems. We try to visualize the action of an extremal positive map by plotting various two dimensional sections through the set of density matrices.

For this purpose it is useful to transform the map to some standard form. We argue that any positive map can be transformed into a unital and trace preserving form through a product transformation of the corresponding entanglement witness. If the witness lies in the interior of $\mathcal{S}^{\circ}$, which means that it has no zeros, this follows from the proof of a similar result given in [15]. We present an iteration scheme for computing the transformation numerically, and we find in practice in $3 \times 3$ dimensions that it works well even for extremal witnesses and other witnesses lying on the boundary of $\mathcal{S}^{\circ}$. We find numerically that the unital and trace preserving form of a positive map is unique up to unitary product transformations.

We present plots related to two different extremal entanglement witnesses in $3 \times 3$ dimensions and one in $2 \times 4$ dimensions. The first example is a randomly chosen generic extremal entanglement witness with quadratic zeros found in previous numerical searches. We produce the corresponding positive map $\mathbf{M}: H_{3} \rightarrow H_{3}$, and then plot two dimensional sections in order to illustrate how the image $\mathbf{M} \mathcal{D}_{3}$ lies inside $\mathcal{D}_{3}$.

We then repeat this scheme for a version of the Choi-Lam map, or entanglement witness, which is extremal but highly non-generic, having only quartic zeros. It has three isolated quartic zeros, and one continuous two dimensional set of zeros which are necessarily quartic.

The most important feature of the plots is related to the zeros of the two witnesses. A zero defines a pure state in $\mathcal{D}_{3}$ which is mapped to the boundary of $\mathcal{D}_{3}$. In particular, a generic extremal witness in $3 \times 3$ dimensions, like the one presented here, has nine zeros, defining a simplex in $\mathcal{D}_{3}$ with nine vertices which is mapped to a simplex in $\mathcal{D}_{3}$ with nine vertices touching the boundary of $\mathcal{D}_{3}$ from the inside.

We should emphasize that we have studied here only $3 \times 3$ and $2 \times 4$ dimensions, which are the simplest nontrivial cases. In higher dimensions the symmetric dimensions $m \times n$ with $m=n>3$ are clearly the most interesting. The complexity increases much with the dimension, because the simplex of pure states in $\mathcal{D}_{3}$ corresponding to the quadratic zeros of the extremal witness considered here, becomes a polytope with a number of vertices larger than $m^{2}$ when $m>3$. For example, with $m=n=4$ the minimum number of zeros of a quadratic extremal witness is 20 , as compared to the dimension of $\mathcal{D}_{4}$ which is 15 . With $m=n=5$ the minimum number of zeros is 37 , as compared to the dimension of $\mathcal{D}_{5}$ which is 24 [11.

We believe that the geometrical way of thinking illustrated here may be a fruitful approach when one wants to construct examples of extremal maps and entanglement witnesses. It may be that the increase in complexity with increasing dimension, which is a well known phenomenon, is easier to handle geometrically than by other methods.

\section{Acknowledgments}

We acknowledge gratefully a research grant from The Norwegian University of Science and Technology (Leif Ove Hansen). We thank Erling Størmer for his interest in our studies of extremal positive maps, and in particular his question what they look like, which inspired the present paper. 


\section{A An extremal positive map from $H_{2}$ to $H_{4}$}

We introduce the following $4 \times 4$ matrices.

$$
B_{0}+B_{3}=\left(\begin{array}{cccc}
a_{1} & -\mathrm{i} a_{3} & \mathrm{i} a_{4} & a_{1} \\
\mathrm{i} a_{3} & a_{2} & 0 & \mathrm{i} a_{3} \\
-\mathrm{i} a_{4} & 0 & a_{2} & -\mathrm{i} a_{4} \\
a_{1} & -\mathrm{i} a_{3} & \mathrm{i} a_{4} & a_{1}
\end{array}\right)
$$

with

$$
\begin{gathered}
a_{1}=0.0244482760740412, \quad a_{2}=0.2152770862261020 \\
a_{3}=0.0114377547217477, \quad a_{4}=0.0500075452822933 \\
B_{0}-B_{3}=\left(\begin{array}{cccc}
a_{5} & \mathrm{i} a_{7} & \mathrm{i} a_{8} & -a_{5} \\
-\mathrm{i} a_{7} & a_{6} & 0 & \mathrm{i} a_{7} \\
-\mathrm{i} a_{8} & 0 & a_{6} & \mathrm{i} a_{8} \\
-a_{5} & -\mathrm{i} a_{7} & -\mathrm{i} a_{8} & a_{5}
\end{array}\right)
\end{gathered}
$$

with

$$
\begin{aligned}
& a_{5}=0.0644909685779951, \quad a_{6}=0.1957836691218616, \\
& a_{7}=0.0774551312933996, \quad a_{8}=0.0177155824920755 . \\
& B_{1}=\left(\begin{array}{cccc}
0 & -a_{9}-\mathrm{i} a_{10} & a_{11}-\mathrm{i} a_{12} & -\mathrm{i} a_{13} \\
-a_{9}+\mathrm{i} a_{10} & 0 & -a_{14}-\mathrm{i} a_{15} & a_{11}+\mathrm{i} a_{16} \\
a_{11}+\mathrm{i} a_{12} & -a_{14}+\mathrm{i} a_{15} & 0 & -a_{9}-\mathrm{i} a_{17} \\
\mathrm{i} a_{13} & a_{11}-\mathrm{i} a_{16} & -a_{9}+\mathrm{i} a_{17} & 0
\end{array}\right)
\end{aligned}
$$

with

$$
\begin{aligned}
& a_{9}=0.0363521121932822, \quad a_{10}=0.0276760626964089, \quad a_{11}=0.0094553411157518, \\
& a_{12}=0.0293657267910500, \quad a_{13}=0.0130745578191192, \quad a_{14}=0.1714859526438769(86) \\
& a_{15}=0.0675990471881839, \quad a_{16}=0.0121590711417975, \quad a_{17}=0.0384768416753617 . \\
& B_{2}=\left(\begin{array}{cccc}
0 & -a_{11}-\mathrm{i} a_{12} & -a_{9}+\mathrm{i} a_{10} & \mathrm{i} a_{18} \\
-a_{11}+\mathrm{i} a_{12} & -a_{14} & \mathrm{i} a_{19} & a_{9}-\mathrm{i} a_{17} \\
-a_{9}-\mathrm{i} a_{10} & -\mathrm{i} a_{19} & a_{14} & a_{11}-\mathrm{i} a_{16} \\
-\mathrm{i} a_{18} & -a_{9}+\mathrm{i} a_{17} & a_{11}+\mathrm{i} a_{16} & 0
\end{array}\right)
\end{aligned}
$$

with

$$
a_{18}=0.0082070224528484, \quad a_{19}=0.0424325553291989 .
$$

The $2 \times 2$ matrix

$$
A=\frac{1}{2}\left(\begin{array}{cc}
u+z & x-\mathrm{i} y \\
x+\mathrm{i} y & u-z
\end{array}\right)
$$

is positive when $u>0$ and $u^{2} \geq x^{2}+y^{2}+z^{2}$. In Section 4.4 we discuss the following extremal positive map, which we do not transform to unital and trace preserving form,

$$
\mathbf{M}: A \mapsto B=u B_{0}+x B_{1}+y B_{2}+z B_{3} .
$$




\section{References}

[1] E. Schrödinger, Discussion of probability relations between separated systems

Proc. Camb. Philos. Soc. 31, 555 (1935)

[2] A. Einstein, B. Podolsky, and N. Rosen, Can Quantum-Mechanical Description of Physical Reality Be Considered Complete? Phys. Rev. 47, 777 (1935)

[3] M. Nielsen and I. Chuang, Quantum Computation and Quantum Information Cambridge University Press, Cambridge (2000)

[4] M. Horodecki, P. Horodecki, and R. Horodecki, Separability of mixed states: necessary and sufficient conditions Phys. Lett. A 223, 1 (1996)

[5] M. Barbieri, F. De Martini, G. Di Nepi, P. Mataloni, G.M. D'Ariano, and C. Macchiavello, Detection of Entanglement with Polarized Photons: Experimental Realization of an Entanglement Witness

Phys. Rev. Lett. 91, 227901 (2003)

[6] A. Jamiołkowski, Linear transformations which preserve trace and positive semidefiniteness of operators Rep. Math. Soc. 3, 275 (1972)

[7] M.D. Choi, Positive semidefinite biquadratic forms Linear Algebra Appl. 12, 95 (1975)

[8] E. Størmer, Positive linear maps of operator algebras Acta Math. 110, 233 (1963)

[9] E. Størmer, Positive linear maps of operator algebras Monographs in Mathematics, Springer Verlag, Berlin (2013)

[10] A. Peres, Separability criterion for density matrices Phys. Rev. Lett. 77, 1413 (1996)

[11] L.O. Hansen, A. Hauge, J. Myrheim, and P.Ø. Sollied, Extremal entanglement witnesses arXiv:1305.2385

[12] M. Lewenstein, B. Kraus, J. I. Cirac, and P. Horodecki, Optimization of entanglement witnesses Phys. Rev. A 62, 052310 (2000) 
[13] E. Størmer,

Decomposable linear maps on $C^{*}$-algebras

Proc. Amer. Math. Soc. 86, 402 (1982)

[14] S.L. Woronowicz,

Positive maps of low dimensional matrix algebras

Rep. Math. Phys. 10, 165 (1976)

[15] J.M. Leinaas, J. Myrheim, and E. Ovrum,

Geometrical aspects of entanglement

Phys. Rev. A 74, 012313 (2006)

[16] M.D. Choi and T.Y. Lam,

Extremal positive semidefinite forms

Math. Ann. 231, 1 (1977)

[17] P. Horodecki,

Separability criterion and inseparable mixed states with positive partial transposition Phys. Lett. A 232, 333 (1997) 